This pre-print has been published, please cite as:

Stachl, C., Au, Q., Schoedel, R., Samuel. D. Gosling, Gabriella. M. Harari., Buschek, D., Völkel, S., Schuwerk, T., ... Bühner, M. (2020). Predicting Personality from Patterns of Behavior Collected with Smartphones. Proceedings of the National Academy of Sciences of the United States of America. https://doi.org/10.1073/ pnas. 1920484117

\title{
Behavioral Patterns in Smartphone Usage Predict Big Five Personality Traits
}

\author{
Clemens Stachl ${ }^{1}$, Quay Au ${ }^{2}$, Ramona Schoedel ${ }^{1}$, Daniel Buschek ${ }^{3}$, Sarah Theres Völkel ${ }^{3}$, Tobias \\ Schuwerk $^{4}$, Michelle Oldemeier ${ }^{1}$, Theresa Ullmann ${ }^{2}$, Heinrich Hussmann ${ }^{3}$, Bernd Bischl ${ }^{2}$, \& Markus \\ Bühner $^{1}$
}

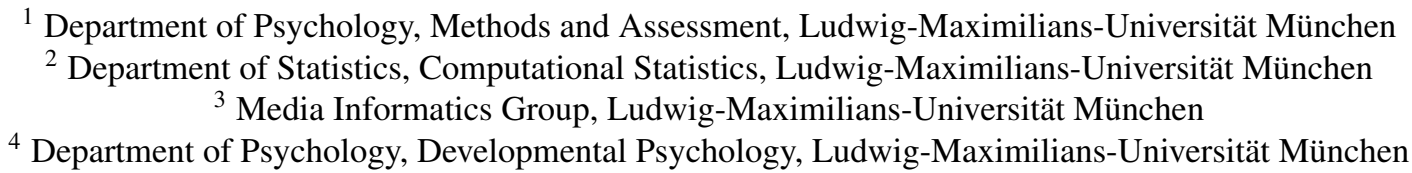

\begin{abstract}
The understanding, quantification and evaluation of individual differences in behavior, feelings and thoughts have always been central topics in psychological science. An enormous amount of previous work on individual differences in behavior is exclusively based on data from self-report questionnaires. To date, little is known about how individuals actually differ in their objectively quantifiable behaviors and how differences in these behaviors relate to big five personality traits. Technological advances in mobile computer and sensing technology have now created the possiblity to automatically record large amounts of data about humans' natural behavior. The collection and analysis of these records makes it possible to analyze and quantify behavioral differences at unprecedented scale and efficiency. In this study, we analyzed behavioral data obtained from 743 participants in 30 consecutive days of smartphone sensing $(25,347,089$ logging-events). We computed variables $(15,692)$ about individual behavior from five semantic categories (communication \& social behavior, music listening behavior, app usage behavior, mobility, and general day- \& nighttime activity). Using a machine learning approach (random forest, elastic net), we show how these variables can be used to predict self-assessments of the big five personality traits at the factor and facet level. Our results reveal distinct behavioral patterns that proved to be differentially-predictive of big five personality traits. Overall, this paper shows how a combination of rich behavioral data obtained with smartphone sensing and the use of machine learning techniques can help to advance personality research and can inform both practitioners and researchers about the different behavioral patterns of personality.
\end{abstract}

Keywords: mobile-sensing, smartphones, behavior, machine learning, interpretable machine learning, big five personality, trait-prediction Word count: 10,621

The systematic assessment and understanding of individual human differences in behavior and experience had already proved useful in first rudimentary tests in ancient China in 2200 BC (Gregory, 2013). Over the time of millennia, this objective has led to the creation of multiple terminologies for the exhaustive description of psychologically relevant individual differences. However, only in the last few decades, the big five personality trait theory (Goldberg, 1990; McCrae \& John, 1992) has emerged as the most widely acknowledged taxonomy in the field of psychological science (De Raad, 2000). The big five taxonomy describes human personality on five broad and relatively stable dimensions: emotional stability, extraversion, openness, conscientiousness, and agreeableness. Furthermore, these dimensions consist of a larger number of underlying facets that describe more narrow aspects of personality. The big five structure has been successfully replicated across several cultures and contexts (Ching et al., 2014; P. Costa Jr, Terracciano, \& McCrae, 2001). Moreover, it has been shown that personality traits are related to impor- tant behaviors and life outcomes such as job performance, well-being, and mental health (Ozer \& Benet-Martínez, 2006; Paunonen \& Ashton, 2001; Roberts, Kuncel, Shiner, Caspi, \& Goldberg, 2007; M. Ziegler et al., 2014). Big five personality traits have also proven useful in many practical applications such as the personalization of advertisements (Matz, Kosinski, Nave, \& Stillwell, 2017), the clinical assessment of psychopathology (P. T. Costa \& Widiger, 1994), and the selection of personnel (Barrick \& Mount, 1991). As personality traits are thought to manifest themselves in behavior, researchers have aimed to investigate which behaviors are most indicative of individual personality trait levels. However, traditionally, it has proven difficult to collect valid data about individual differences in human behavior without the use of self-report questionnaires (Baumeister, Vohs, \& Funder, 2007; Furr, 2009). Only recently, the increased availability of rich data about human behavior in the form of social network and website profiles has provided new perspectives for the investigation of individual differences (Wilson, Gosling, \& 
Graham, 2012). In particular, these new data allow scientists to conduct research using larger datasets of higher quality and finer data-granularity (Mahmoodi, Leckelt, Zalk, Geukes, \& Back, 2017). Using this potential, some researchers have demonstrated that online records of human behavior can be informative about intimate traits such as personality, sexual orientation, and political attitudes (Gladstone, Matz, \& Lemaire, 2019; Kosinski, Stillwell, \& Graepel, 2013; Park et al., 2015; Schwartz et al., 2013; Youyou, Kosinski, \& Stillwell, 2015). See Azucar, Marengo, and Settanni (2018) for a recent review of personality prediction from social media data.

However, beyond the usage of pre-existing data from social networks, it has been more difficult to investigate associations of personality and behavior and to make personality judgments about people who do not provide much data online (e.g., are inactive or not registered on social networks). As peoples' activity is shifting from traditional social network platforms (e.g., Facebook) to mobile-first social networks (e.g., Instagram, Anderson \& Jiang, 2018), it is a logical and necessary step to use mobile phones for the collection of behavioral data (Miller, 2012; Wrzus \& Mehl, 2015). The mobile phone has become the most popular device with global mobile cellular subscription rates exceeding $70 \%$ and $100 \%$ (more subscriptions than people) in developed countries (Union, 2018). Popular due to their enormous versatility, modern smartphones can be used for listening to music, using social networks, navigating new environments, shopping, photography, and of course communication. This channeling of behavior trough a single device has led to an enormous increase in personal

Clemens Stachl, Department of Psychology, Psychological Methods and Assessment, Ludwig-Maximilians-Universität München; Quay Au, Department of Statistics, Computational Statistics, Ludwig-Maximilians-Universität München; Ramona Schoedel, Department of Psychology, Psychological Methods and Assessment, Ludwig-Maximilians-Universität München; Daniel Buschek, Media Informatics Group, Ludwig-MaximiliansUniversität München; Sarah Theres Völkel, Media Informatics Group, Ludwig-Maximilians-Universität München; Tobias Schuwerk, Department of Psychology, Developmental Psychology, Ludwig-Maximilians-Universität München; Michelle Oldemeier, Department of Psychology, Psychological Methods and Assessment, Ludwig-Maximilians-Universität München; Theresa Ullmann, Department of Statistics, Computational Statistics, LudwigMaximilians-Universität München; Heinrich Hussmann, Media Informatics Group, Ludwig-Maximilians-Universität München; Bernd Bischl, Department of Statistics, Computational Statistics, LudwigMaximilians-Universität München; Markus Bühner, Department of Psychology, Psychological Methods and Assessment, LudwigMaximilians-Universität München

Correspondence concerning this article should be addressed to Clemens Stachl, Leopoldstr. 13, 80802 München, Germany, EU. E-mail: clemens.stachl@psy.lmu.de convenience and effectiveness, and has transformed mobile phones into highly personal devices. Thus, the capability to collect data about these activities allows for the investigation of systematic variations in human behaviors, feelings, and preferences at unseen scale and with unprecedented precision (Harari et al., 2016). In addition to the traceability of active behaviors, sensors in smartphones also allow for the passive collection of situational data (e.g., environmental illumination and noise, GPS etc.), making them especially suited for the investigation of individual differences in behavior across different situations (Harari, Gosling, Wang, \& Campbell, 2015). Finally, it can be assumed that behavioral data from smartphones have a high ecological validity because they can be collected automatically and unobtrusively in the field.

Behavior and Personality. Only few studies have investigated how personality traits are manifested in smartphone usage. Most of theses studies have primarily focused on associations of communication behavior with levels of big five personality traits. In that regard, extraversion was found to be positively related to the frequency and duration of calls, the number and frequency of text messaging, the frequency and duration of conversations, and the frequency of communication app usage on smartphones (Chittaranjan, Blom, \& Gatica-Perez, 2013; Harari et al., 2019; Montag et al., 2014, 2015; Stachl et al., 2017).

In addition to communication behavior, some of these studies have also investigated associations of big five personality traits with the usage of other applications (apps) (Chittaranjan et al., 2013; Kim, Briley, \& Ocepek, 2015; Stachl et al., 2017). Kim et al. (2015) found higher levels of self-reported conscientiousness to be negatively associated with the use of e-commerce related applications (e.g., shopping, banking). In their study, Chittaranjan et al. (2013) reported negative correlations between extraversion scores and app usage related to gaming, web-browsing, and camera usage. Additionally, they found the usage of office-related applications to be positively related to levels of extraversion. Furthermore, they reported a number of negative correlations between agreeableness and the usage of office-, internet-, mail-, calendar-, and media applications. Additionally, conscientiousness was found to be negatively related to the usage of media applications. Finally, emotional stability showed negative associations with apps related to office activities. Stachl et al. (2017) found that higher levels of conscientiousness were associated with a decreased usage of gaming applications. Furthermore, they found that higher levels of agreeableness were associated with increased usage of apps related to transportation. Finally, they reported that on average, the usage of photography-related applications was increased for people with higher scores in extraversion.

While most of these studies highlight the potential of smartphone-sensing methods for research, their results are 
also limited in generalizability as analyses have mostly been performed on small samples without cross-validation. Furthermore, the mixed pattern of previous findings hints at previously reported differences between self-reported and actually observed behaviors (Ellis, 2019; Gosling, John, Craik, \& Robins, 1998). To overcome these limitations, we aim to include a broad range of behaviors which can nowadays be sensed via smartphones. This approach builds on previous studies which have reported on behavioral manifestations of personality traits in various types of behavior (in non-digitized contexts).

Music preferences for example, have repeatedly been linked to individual personality traits. Most importantly, the personality dimensions of extraversion and openness have been repeatedly associated with preferences for specific types of music. Langmeyer, Guglhör-Rudan, and Tarnai (2012) found that on average, people with higher scores in extraversion prefer conventional and energetic music. People scoring higher on the dimension of openness were found to prefer more sophisticated, complex-, non-conventional and classical music (Dunn, Ruyter, \& Bouwhuis, 2012; Langmeyer et al., 2012; Nave et al., 2018). Beyond genre-based analyses, Greenberg et al. (2016) loosely linked individuals' preference for three musical attribute dimensions to individual personality traits. In addition to correlational results of earlier works, latest studies using machine learning suggest that personality trait scores can partially even be predicted from self-reported music preferences and Facebook likes (Nave et al., 2018).

Individual differences in personality have also been found with regard to the temporal distribution of behaviors throughout the day. In the field of sleep-research, scientists have investigated associations of various self-reported diurnal activities with levels of big five personality traits (Randler, Baumann, \& Horzum, 2014). According to meta-analytical findings, conscientiousness has most robustly been related to a preference for morning activities (Tsaousis, 2010). Whereas Duggan, Friedman, McDevitt, and Mednick (2014) found low emotional stability to be related to early wake-up times and poor sleep quality, Randler et al. (2014) found extraversion to be positively related to a preference for nightly activity.

A relatively new area of research for the investigation of individual differences is the analysis of data about individual mobility and physical activity. Although little research exists about possible associations between personality traits and individual mobility patterns (Mehrotra et al., 2017; Schoedel et al., 2018), affective states have been successfully linked to reduced physical activity and gyration (Lathia, Sandstrom, Mascolo, \& Rentfrow, 2017; Saeb, Lattie, Schueller, Kording, \& Mohr, 2016; Saeb et al., 2015). Differences in the experience of negative affect in turn have been found to be related to the personality dimensions of emotional stability and extraversion for a long time (P. T. Costa \& McCrae, 1980).
The Prediction of Personality. While digital footprints from social media data have successfully been used to predict personality traits (Settanni, Azucar, \& Marengo, 2018), only few studies have started to consider the usage of data from smartphones to achieve this goal (Chittaranjan et al., 2013; De Montjoye, Quoidbach, Robic, \& Pentland, 2013; Mønsted, Mollgaard, \& Mathiesen, 2018; Schoedel et al., 2018). Earlier studies have reported relatively high performance in the prediction of big five personality traits from smartphone usage, despite limited data and rather small sample sizes (Chittaranjan et al., 2013; De Montjoye et al., 2013). Later studies reported larger sample sizes but lower predictive performances for both the big five personality dimensions (Mønsted et al., 2018) and the personality dimension of sensation seeking (Schoedel et al., 2018). However, as demonstrated by Mønsted et al. (2018), results of earlier works most likely have been overly optimistic, due to model over-fitting (Chittaranjan et al., 2013; De Montjoye et al., 2013). Mønsted et al. (2018) concluded that only the factor of extraversion could be reliably predicted from smartphone usage parameters. The authors argued that this is related to phones being mainly used for communication, which in turn, is related to extraversion (e.g., Montag et al., 2015). Although this logic seems intuitive, we argue that the predictability of other personality dimensions might not have been fully evaluated, as Mønsted et al. (2018) almost exclusively used predictors related to individual communication behavior. First, smartphones can be used for various purposes and are therefore not limited to making calls and sending messages. Second, according to big five personality theory, personality should be reflected in the broad spectrum of an individual's behaviors (Funder, 2001). Hence, it is likely that the utilization of behavioral patterns across a range of activities will make it possible to predict other big five personality traits, besides extraversion. This notion is also supported by a recent meta-analysis on personality prediction from social media data, showing that on average, a combination of different data types increased prediction performance in previous studies (Settanni et al., 2018).

Taken together, few and inconclusive results from past research motivate our efforts to more intensively explore the possibility of personality trait prediction from a wide range of objective behaviors, collected via smartphone-sensing. We first extract behavioral predictors from a wide range of daily behaviors. Second, we use these variables to predict self-reported big five personality scores on factor and facet levels with a machine learning approach. During model-evaluation, we apply appropriate nested cross-validation procedures and compare the prediction performance of different models against a common guessing-baseline. Third, we inspect the mostpromising machine learning models and investigate which patterns of behavior are most important for the prediction of each individual personality trait dimension. Finally, we 
discuss the influence of these variables with regard to previous findings and highlight opportunities for further, confirmatory research.

\section{Method}

The dataset used in this study was composed of three distinct datasets, collected in three separate studies within the context of the PhoneStudy research project at the LudwigMaximilians-Universität München (LMU) (Stachl et al., 2018). Although, small parts of the data have been used in other publications (Schoedel et al., 2018; Schuwerk, Kaltefleiter, Au, Hösl, \& Stachl, 2018; Stachl et al., 2017), the joint dataset of common parameters has not been analyzed before. Procedures for all three studies have been approved by institutional review boards and have been conducted according to EU-laws and ethical standards. See Table 1 for an overview of the included datasets. Although it is not possible to provide the raw data files to the research community (due to unsolved privacy implications), we provide the final dataset of aggregated variables, the code for variable-extraction, preprocessing, predictive-modeling and a data dictionary, including descriptions and additional explanations as additional resources in the project's repository (Stachl et al., 2019).

\section{Participants}

We recruited a combined number of 743 participants from the academic population at LMU, from forums, social media, blackboards, flyers, and direct recruitment in the streets of Munich, Germany, between September 2014 and January 2018. During the pre-processing of the data, the sample was reduced to 624 participants. First we excluded participants with less than 15 days of logging data (29). Furthermore, 39 participants that did not use any apps and 51 participants that did not complete the necessary questionnaires were excluded from further analysis.

The final sample consisted of 377 women, 243 men, and 4 participants that chose not to disclose their gender. The participants average age was $23.56(\mathrm{SD}=6.63)$. Furthermore, most of the participants $(91 \%)$ had successfully completed A-levels and $20 \%$ reported a finished university degree. See Table 2 and the project's repository for more descriptive statistics. All subjects participated willingly and gave consent prior to their participation in the study. The participants could withdraw their participation at any time during the ongoing data collection period. Additionally, they could demand the deletion of their data for as long as their re-identification was possible (during ongoing data collection). Dependent on the respective study, different rewards for participation were provided to the participants. In study 1 , an individualized personality profile and $30 €$ or course credit ( $3 \mathrm{~h}$ ) for students were offered. In study 2 , participants were rewarded $25 €$ to $35 €$, dependent on their participation in the experience sampling scheme of the study (see, Schuwerk et al., 2018). Additionally, the participants could enter a lottery to win a smartphone (value $400 €)$. In study 3 , individualized personality and user activity feedback and optional course credit were provided to the participants (Schoedel et al., 2018) as reimbursement. Similar to study 2 , participants could also enter a lottery to win $10 \times$ $50 €$.

\section{Self-Reported Personality Measures \& Demographics}

Big five personality dimensions were measured with the German version of the Big Five Structure Inventory (BFSI) on factor and facet level, via 30 subscales. The BFSI was used due to its good psychometric properties and relatively short test-duration (Arendasy, 2009). The test consists of 300 items (adjectives and short phrases) and measures the Big Five personality dimensions (emotional stability, extraversion, openness to experience, conscientiousness, and agreeableness) on five factors and 30 facets. Agreement to single items was stated on a four-point Likert scale ranging from untypical for me to rather untypical for me to rather typical for me to typical for me. In contrast to more common personality inventories such as the NEO-PI-R or the more current version NEO-PI-3 (McCrae, Costa, \& Martin, 2005), the BFSI uses adjectives and short statements and has been developed using item response theory rather than classical test theory. The authors report psychometric benefits over other similar questionnaires due to conformity with the partial credit model (Masters, 1982). In study 1, questionnaires were administered via desktop computers. In study 2 , questionnaires were administered two times, once via desktop computer and once via smartphone. In this study we used the lab version scores from study 2 . In study 3 , all questionnaires were provided via smartphone to the participants. In all three studies identical personality items and answer formats were used. Descriptive statistics including confidence intervals of internal consistencies for all scales are provided in Table 2.

In addition to the personality scores we collected age, gender, and the level of completed education. Gender was recorded dichotomously, age was measured as the birth-date and later converted to age in years. The level of education was subdivided in five categories from no education to compulsory education to vocational training to A-levels to finished university degree. Please note that for data from study 3 , a sixth category finished PhD/habilitation was re-coded into finished university degree. In addition to measures used in this manuscript, participants completed a number of other questionnaires that were used for other research projects in the respective studies. In the present study, we only included measures that had been collected across all studies and that were relevant for the 
Table 1

Datasets used in this study

\begin{tabular}{lccll}
\hline Data Set & N & Collection Period & \multicolumn{1}{c}{ Events } & \multicolumn{1}{c}{ References } \\
\hline Study 1 & 137 & $09 / 2014-08 / 2015$ & $1,776,317$ & Stachl et al. (2017), Harari et al. (2019) \\
Study 2 & 242 & $08 / 2016-08 / 2017$ & $4,236,022$ & Schuwerk et al. (2018) \\
Study 3 & 245 & $10 / 2017-01 / 2018$ & $19,334,750$ & Schoedel et al. (2018) \\
Combined & 624 & $09 / 2014-01 / 2018$ & $25,347,089$ & \\
\hline
\end{tabular}

Note. Overview of datasets used in this study. Events represent the total amount of logged activities across all participants and days before variable extraction.

research question at hand. Although additional measures will not be further described here, more information can be found in the respective online-repositories and articles (Harari et al., 2019; Schoedel et al., 2018; Schuwerk et al., 2018; Stachl et al., 2017).

\section{Behavioral Data from Mobile Sensing}

We used the PhoneStudy smartphone research app for the Android mobile operating system to collect behavioral data from the participants' privately owned smartphones. This app has been continuously developed at the Ludwig-MaximiliansUniversität München since September 2013, to effectively and unobtrusively record what people do with their phones, when, where, and how. In contrast to traditional methods of behavioral observation (e.g., lab-based behavioral observation), the usage of the PhoneStudy app enabled us to continuously record a wide range of parameters related to participants' behavior, as they occurred in daily life. Initially, activities were recorded in the form of time-stamped logs of events. Those included events related to calling, messaging, GPSlocation, app starts/installations, screen de-/activations, flight mode de-/activations, Bluetooth connections, booting events, played music, battery charging status, photo and video events (no actual photos or videos), and connection events to wireless networks (WiFi). Additionally, the character-length of messages and technical device characteristics were collected. Irreversibly hash-encoded versions of contacts and phonenumbers were collected in order to enable the distinction of contacts while preventing the possibility of re-identification. Information such as names, phone numbers and contents of messages, calls etc. were not recorded at any time.

\section{Procedures}

Study procedures were somewhat different with regard to the respective study (Schoedel et al., 2018; Schuwerk et al., 2018; Stachl et al., 2017). However, common to all three studies, big five personality trait levels were collected with the BFSI at least once and naturalistic smartphone usage in the field was automatically recorded over a period of 30 days. In all three studies, the collected user data was regularly transferred to our server using SSL-encryption, during times participants were connected to a wireless network. Data was stored in encrypted form on the servers. In study 1, participants did not have to complete any tasks during data collection in the field. In study 2, participants had to answer experience sampling questionnaires during the data collection period on their smartphones (Schuwerk et al., 2018). Participants in study 3 also had to complete the demographic and BFSI personality questionnaires via smartphone at a convenient time (Schoedel et al., 2018). In all three studies, participants were automatically reminded to activate location services, in the case they had turned them off. Finally, at the end of mobile data collection, participants were instructed to contact the research staff in order to receive compensation (studies 1-3) and to return for the final lab session (study 2). More details about the procedures of the respective studies are available in the respective research articles (Schoedel et al., 2018; Schuwerk et al., 2018; Stachl et al., 2017).

\section{Data Analysis}

The final data set consisted of 1821 predictor variables and 35 ( 5 factors and 30 facets) personality criteria. Gender, age, and education were solely used for descriptive statistics and were not included as predictors in any of the models. Please note that although it is not possible to provide the raw data files to the research community (due to unsolved privacy implications), we provide the final dataset of aggregated variables, the syntax for variable-extraction, pre-processing, predictivemodeling and a data dictionary, including descriptions and additional explanations as supplementary files in the project's osf-repository (Stachl et al., 2019).

Pre-Processing. Before the machine learning analysis, we applied a series of pre-processing steps with regard to the characteristics of the specific data-types (e.g., location data, appusage, communication etc.). For texting and calling behaviors, 
apparent logging-induced duplicate entries were removed to increase the data quality (also see Harari et al., 2019). In order to create variables about categorical app-usage, we first enriched the app-usage data with additional parameters from the Google Play Store, using web-scraping (Google, 2019). This additional data provided information about the type, the price and the popularity of an app. However, as the appcategories from the Google Play Store proved to be inaccurate for many apps (e.g., "dating" apps being labeled as "lifestyle" apps) and because some apps used by our participants were not available in the Google Play Store, we manually assigned each app to new, semantic categories. This was done in an iterative fashion and was cross-checked by three researchers. The full table with all observed apps and the assigned categories is available as a supplemental file in the project's repository (Stachl et al., 2019).

We enriched the data about participants' music listening behavior with information from the Spotify API. In contrast to previous approaches (Greenberg et al., 2016; Langmeyer et al., 2012; Nave et al., 2018), this approach allowed us to create rather objective quantifications of participants' music preferences. In particular, we retrieved metadata with the track search API (Spotify, n.d.-a), including the audio features object (Spotify, n.d.-b) (e.g., stylistic characteristics such as "acousticness" or "danceability"). Further details on these variables are available at the given links to the Spotify API. The API calls required specifying a combination of album, artist, and track title. To do so, we used the track information from the PhoneStudy-app. However, the logged track information can be incorrect in some cases, for example, if the track file played by a participant (locally) had incorrect or missing metadata (e.g., spelling mistake in the title, album missing). To still retrieve information in these cases, we repeatedly called the Spotify API using several heuristics: First, we used the full available track information without modifications. If no result was found, we removed special characters (e.g., punctuation) and "tags" in those fields (i.e. characters between brackets, e.g., "[Bonus CD]"). If this was still unsuccessful, we subsequently tried further searches, such as searching without album and with "split" artist field (sometimes artist data incorrectly contained a list of artists instead of only the main artist). These heuristics were developed and manually checked on a test set of incorrect track information, compiled from our logged data. In this way, we improved coverage of successfully retrieved data without compromising on the quality. For example, we found that searching only via track title was not acceptable as it resulted in many incorrect matches. In total, we retrieved metadata for $76 \%(63,648)$ of all unique logged tracks. After we enriched the music listening records with additional parameters from the Spotify API, we manually checked whether the retrieved music parameters were correctly matched to the listened interpret-title-album triples. Consequently, the remaining music data was further reduced to songs that were listened to for more than 20 seconds and songs with a speechiness value of less than 0.6. We implemented these cutoffs to exclude skipped songs, audio-books, and podcasts for more accurate parameter-estimation in the consequent step of variable-extraction.

For location data, we used the Google Elevation API (Google, 2018) to assign levels of ground elevation to longitude-latitude combinations.

Finally, after the completion of this first step of pre-processing, the complete set of predictor variables was extracted from the raw dataset (see subsection Variable Extraction). In this second step of pre-processing, GPS-related variables from five participants that used apps deliberately designed to create fake GPS positions, were imputed with missing values ${ }^{1}$. Consequently, missing values of predictors with regard to usage frequencies and durations were imputed with zeros, as missing values would in this case indicate no usage. Furthermore, we excluded variables with less than $2 \%$ unique values - as they would not add much information to the modeling process and would cause our models to crash in some resamplinginstances. In this step we reduced the initial number of 15,692 variables to the final dataset of 1821 variables. This enormous reduction in variables is related to a high number of variables with little to no variance (e.g., apps used only by one participant). Finally, we eliminated extreme outliers by excluding data points that are unreasonably far (greater than 100 times the median absolute deviation) from the sample median. This was done to minimize the impact of possible logging errors on the modeling process.

In addition to pre-processing prior to the analyses, a third step of data-dependent pre-processing was embedded within the resampling procedures of the machine learning models. This process is described in the section Machine Learning. More details about the pre-processing of the data can be found in the respective code files in the osf-repository (Stachl et al., 2019).

Variable Extraction. In total, we extracted 15,692 variables from the raw dataset. In relation to previous studies, the extracted variables roughly corresponded to the behavioral categories of communication, app-usage, music consumption, general day- and nighttime activity (day- and nighttime dependency was treated as a distinct category in the analyses), and mobility. Note that no variables with regard to day and night difference in activity were computed for music listening behavior. Besides common estimators (e.g., arithmetic mean, standard deviation, sum etc.), we also computed more complex variables containing information about the irregularity, the entropy, the similarity, and the temporal correlation of

\footnotetext{
${ }^{1}$ After closer inspection we concluded that this behavior was likely caused by participants creating fake GPS positions while playing Pokémon Go.
} 
behaviors. These additional variables provided information about specific data types (e.g., mobility data) and aimed to grasp behavioral structures within-person and across time. In particular, this allowed us to consider temporal variation in the data, without the need to use more complex time-series models. Furthermore, due to the large amount of data that made hand checking the calculated variables unfeasible, we used robust estimators (e.g., Huber M-Estimator, Huber, 1981) for most variables (except for call and SMS variables that were checked manually). Details about the calculation of variables, the full set of extracted variables, and a detailed overview of all sensed data are provided in the project's repository (Stachl et al., 2019).

Machine Learning. We trained machine learning models for the prediction of all personality factor and facet variables. For model-benchmarking, we compared the predictive performance of elastic net regularized linear regression models (Zou \& Hastie, 2005) with those of non-linear tree-based random forest models (Breiman, 2001) and a baseline model. The baseline model simply predicted the mean of the respective training set for all cases in a test-set. We chose these models due to their ability to cope with $P>>N$ problems (e.g., few cases, many predictors) and because they represent standard models in the machine learning community. Additionally, the usage of random forest models allowed us to harness non-linear predictor-effects in the models.

In order to evaluate the predictive performance of our models we implemented hyperparameter tuning and pre-processing in a nested resampling approach (Bischl, Mersmann, Trautmann, $\&$ Weihs, 2012). This approach allowed us to test for optimal model settings while keeping the training and testing parts of the data strictly separated. Thus, this approach helps to avoid algorithmic over-fitting as it allows the model to only consider the characteristics of the respective training set fold for data-dependent pre-processing decisions. Specifically, we used an inner loop for model optimization and an outer resampling loop for performance evaluation. In the inner resampling loop, we performed additional pre-processing and hyperparameter tuning, using 5-fold cross-validation. In the outer loop, the expected predictive-performance on new, unseen data was estimated using 10 times repeated 10-fold cross-validation. Within each fold of both cross-validation schemes, constant variables were dropped and missing values of numeric and integer variables were either imputed with two times the maximum of the remaining values (random forest) or with the median (elastic net). We used the two times maximum strategy to label missings as distinct cases in order to allow random forest models to effectively use that information in the modeling process. Median imputation for elastic nets was chosen as it was expected that this approach would not greatly influence the models. Predictors in the elastic net models were additionally centered and scaled within each fold of the inner cross-validation loop. In contrast to simpler linear models (e.g., linear regression), more flexible non-linear models (e.g., random forests) use hyperparameters that can be tuned for optimal model performance. We used a random search approach for the tuning of hyperparameters in both model-types. In random forest models, we tuned the number of randomly considered split variables and the minimum number of observations in a node to be considered (min.node.size) for a split (Wright \& Ziegler, 2017). In elastic net models, we tuned the regularization parameter $\lambda$ and the regularization-mixing parameter $\alpha$ (J. Friedman, Hastie, \& Tibshirani, 2010).

We evaluated the predictive performance of the models based on the Pearson correlation $(r)$ between the predicted values and the person-parameter trait-estimates from the selfreported values of the respective personality trait variables. Additionally, we considered the root mean squared error $(R M S E)$ and the coefficient of determination $\left(R^{2}\right)$ as measures of predictive performance:

$$
\begin{gathered}
\text { RMSE }=\sqrt{\frac{1}{n} \sum_{i=1}^{n}\left(y_{i}-\hat{y}_{i}\right)^{2}} \\
R^{2}=1-\frac{\sum_{i=1}^{n}\left(y_{i}-\hat{y}_{i}\right)^{2}}{\sum_{i=1}^{n}\left(y_{i}-\bar{y}\right)^{2}}
\end{gathered}
$$

We computed performance measures within each fold of the cross-validation procedure and averaged across all folds within a single prediction model (e.g., for extraversion). We considered models as predictive if they showed a positive Pearson correlation between the predicted and actual personality scores, if the mean $R^{2}$ metric was greater than zero, and if the RMSE was lower in a prediction model than in the comparable baseline model.

Interpretable Machine Learning. Machine learning models are often referred to as black-box models, due to their high complexity and their often low explainability (Pargent \& Albert-von der Gönna, 2018; Yarkoni \& Westfall, 2017). To address this problem, a series of methods have been developed to better understand how particular models work (Breiman, 2001; Fisher, Rudin, \& Dominici, 2018). Furthermore, researchers in the fields of statistics and machine learning continue to invent tools to better understand how black-box models work (Guidotti et al., 2018; Molnar, Casalicchio, \& Bischl, 2018). For the prediction models in our study, we computed permutation variable importance values to measure the impact of single variables and semantically grouped variables (Casalicchio, Molnar, \& Bischl, 2019; Gregorutti, Michel, \& Saint-Pierre, 2015). With this method, variable importance is measured by the decrease in the model's prediction performance after permuting one single variable 
(or a group of variables). The motivation behind this is that by shuffling the variables and thus destroying the connection to the target variable, unimportant variables should not influence the prediction performance, but important variables should. Furthermore, for the random forest models, we visualized the influence of single variables on the prediction with accumulated local effect plots (ALE-plots, Apley, 2016). ALE-plots are a method to visualize the effects of individual predictor variables by showing how they affect the prediction of the model on average.

Software. Due to the high computational load of the machine learning analyses, we parallelized the computations on the Linux -Cluster of the LRZ-Supercomputing Center, in Garching near Munich, Germany, EU. For computations on the cluster, R-version 3.5.0 was used. We used R 3.5.2 for all other analyses. We used the fxtract package (Au, 2019) for variable extraction from the raw data. Furthermore, we used the mlr (Bischl et al., 2016), mlrCPO (Binder, 2018), and caret (Jed Wing et al., 2018) packages for pre-processing. For machine learning we additionally used the glmnet (J. Friedman et al., 2010), and ranger (Wright \& Ziegler, 2017) packages. Computational parallelization was enabled through the parallel (R Core Team, 2018), parallelMap (Bischl \& Lang, 2015), and batchtools (Lang, Bischl, \& Surmann, 2017) packages. More details on all used packages can be found in the R-syntax files in the project's repository (Stachl et al., 2019).

\section{Results}

\section{Descriptive Statistics}

First, we computed a series of descriptive statistics in order to portrait characteristics of the dataset and correlations between psychometric and demographic variables. Across the three studies, the participants used a total number of 4,857 different apps, with each participant having used 13.10 (SD $=5.93$ ) distinct apps per day, on average. Whereas the most popular app - WhatsApp was on average used 40.62 (SD = 40.32) times per day, per participant, the more traditional call functionality was only used $1.24(\mathrm{SD}=1.58)$ times per participant, per day, on average. Out of 624 participants, 355 , at least once listened to music on their smartphones, during the course of the study. Furthermore, participants on average listened to 11.13 ( $\mathrm{SD}=12.17$ ) songs per day. Based on GPS data, participants on average covered a robust daily radius of gyration of $6,204(\mathrm{SD}=14,800)$ meters. On an average day, participants used their phone at 23:41 for the last time and at 7:55 for the first time. Considering this, participants on average showed a statistically robust nightly period of inactivity of 7.21 hours ( $\mathrm{SD}=2.41)$. In terms of more general phone usage, participants activated and unlocked the screen of their smartphones an average number of $47.73(\mathrm{SD}=30.86)$ times per day. Furthermore, participants were connected to WiFi networks for an average duration of $4.81(\mathrm{SD}=2.76)$ hours, per day. Descriptive statistics of demographic and personality trait variables are provided in Table 2, correlations between big five personality traits and demographic variables are provided in Table 3. Furthermore, we calculated pairwise Spearman correlations with $95 \%$ bootstrapped percentile confidence intervals between all extracted, behavioral variables and all personality trait variables. Descriptive correlations between all personality trait variables and all behavioral variables are available in the project's repository (Stachl et al., 2019).

In the dataset, we found small correlations between gender and the self-reported level of extraversion ( $r=-.09, C_{95 \%}=[-$ $.17,-.01])$ and between gender and emotional stability $(r=.27$, $\left.C I_{95 \%}=[.18, .33]\right)$. Hence, male gender was associated with lower self-reported levels of extraversion and higher levels of emotional stability. Additionally, we observed substantial correlations between big five personality factors, for example between extraversion and openness $\left(r=.49, C I_{95 \%}=[.43\right.$, .55]) and between extraversion and emotional stability $(r=$ $\left..46, \mathrm{CI}_{95 \%}=[.38, .52]\right)$.

\section{Personality Trait Prediction with Behavioral Patterns}

The results of our analyses show that levels of big five personality traits were successfully predicted from digital records of smartphone usage for the majority of factors and facets. Furthermore, our results suggest differences in how well single personality-dimensions were predicted from the dataset, see Figure 1 and Table 4 . The results also show that the non-linear random forest models on average outperformed the linear, elastic net models in both prediction-performance and the number of successfully predicted criteria. Behavioralpattern plots and semantic importance indicators in Figure 2 provide patterns of variable importance across all models. Several patterns are visible: app-usage behavior had an influence on most of the prediction models, while having the least influence on models for extraversion dimensions. Mobility variables on the contrary, had the least impact on the prediction performance of models. Furthermore, variables from the remaining semantic categories were mostly impactful for prediction models of specific personality dimensions.

Variable importance measures for all successful models are provided for single variables and semantic categories in the project's repository (Stachl et al., 2019). In the following we will report on prediction performances with regard to the respective personality traits. Selected ALE-plots are visible in Figure 3. For reasons of clarity and due to the model's superiority in prediction, we will only report performance metrics from the random forest models in the text. However, 
Table 2

Descriptive statistics of personality and demographic variables.

\begin{tabular}{|c|c|c|c|c|c|c|c|}
\hline & mean & sd & median & $\min$ & $\max$ & range & alphaCI \\
\hline age & 23.56 & 6.63 & 22 & 12 & 72 & 60 & - \\
\hline education & 4.09 & .61 & 4 & 1 & 5 & 4 & - \\
\hline (ES) Emotional Stability & -.02 & .72 & -.01 & -2.24 & 2.52 & 4.76 & {$[.93, .94]$} \\
\hline (E) Extraversion & -.01 & .74 & -.04 & -2.44 & 2.13 & 4.58 & {$[.95, .96]$} \\
\hline (O) Openness & -.05 & .71 & -.11 & -2.00 & 2.12 & 4.12 & {$[.93, .94]$} \\
\hline (C) Conscientiousness & -.09 & .74 & -.14 & -2.28 & 2.29 & 4.57 & {$[.95, .96]$} \\
\hline (A) Agreeableness & -.05 & .76 & -.14 & -2.11 & 2.64 & 4.75 & {$[.93, .94]$} \\
\hline (ES1) Carefreeness & .14 & 1.30 & .27 & -4.30 & 4.30 & 8.60 & {$[.86, .89]$} \\
\hline (ES2) Equanimity & .58 & 1.07 & .49 & -2.61 & 5.02 & 7.63 & {$[.62, .70]$} \\
\hline (ES3) Positive mood & .95 & 1.43 & .96 & -5.78 & 5.59 & 11.38 & {$[.78, .83]$} \\
\hline (ES4) Self consciousness & .65 & 1.18 & .78 & -3.57 & 3.90 & 7.47 & {$[.80, .84]$} \\
\hline (ES5) Self control & .65 & 1.00 & .74 & -3.55 & 5.15 & 8.70 & {$[.79, .84]$} \\
\hline (ES6) Emotional robustness & .66 & 1.19 & .79 & -3.78 & 5.53 & 9.30 & {$[.85, .88]$} \\
\hline (E1) Friendliness & 1.43 & 1.28 & 1.37 & -1.91 & 5.41 & 7.33 & {$[.76, .81]$} \\
\hline (E2) Sociableness & 1.29 & 1.76 & 1.28 & -4.50 & 5.64 & 1.14 & {$[.86, .89]$} \\
\hline (E3) Assertiveness & .44 & 1.37 & .47 & -3.35 & 5.61 & 8.96 & {$[.80, .84]$} \\
\hline (E4) Dynamism & 1.21 & 1.59 & 1.15 & -4.11 & 5.94 & 1.05 & {$[.86, .89]$} \\
\hline (E5) Adventurousness & .43 & 1.49 & .55 & -4.40 & 5.27 & 9.67 & {$[.81, .85]$} \\
\hline (E6) Cheerfulness & 1.97 & 1.66 & 1.85 & -3.23 & 6.09 & 9.32 & {$[.79, .84]$} \\
\hline (O1) Openness to imagination & 1.27 & 1.39 & 1.30 & -2.29 & 5.33 & 7.62 & {$[.80, .84]$} \\
\hline (O2) Openness to aesthetics & .38 & 1.29 & .29 & -4.55 & 4.61 & 9.16 & {$[.90, .92]$} \\
\hline (O3) Openness to feelings & 2.02 & 2.08 & 2.00 & -5.65 & 6.04 & 11.69 & {$[.84, .87]$} \\
\hline (O4) Openness to actions & 1.35 & 1.40 & 1.44 & -2.75 & 5.42 & 8.16 & {$[.85, .88]$} \\
\hline (O5) Openness to ideas & 1.66 & 1.43 & 1.58 & -1.63 & 5.51 & 7.15 & {$[.88, .90]$} \\
\hline (O6) Openness to value \& norm & .91 & 1.03 & .94 & -3.54 & 4.86 & 8.41 & {$[.86, .89]$} \\
\hline (C1) Competence & .84 & 1.21 & .72 & -3.32 & 5.66 & 8.99 & {$[.83, .86]$} \\
\hline (C2) Love of order & 1.10 & 1.55 & 1.16 & -4.34 & 5.67 & 1.01 & {$[.79, .83]$} \\
\hline (C3) Sense of duty & 1.94 & 1.41 & 1.78 & -1.59 & 5.50 & 7.10 & {$[.84, .88]$} \\
\hline (C4) Ambition & 1.83 & 1.67 & 1.58 & -2.39 & 5.86 & 8.25 & {$[.83, .86]$} \\
\hline (C5) Discipline & 1.46 & 1.46 & 1.50 & -3.61 & 5.75 & 9.36 & {$[.75, .80]$} \\
\hline (C6) Caution & 1.53 & 1.34 & 1.45 & -2.51 & 5.75 & 8.26 & {$[.80, .84]$} \\
\hline (A1) Willingness to trust & .37 & 1.42 & .19 & -4.14 & 5.42 & 9.56 & {$[.83, .87]$} \\
\hline (A2) Genuineness & 1.00 & .95 & .86 & -1.56 & 4.25 & 5.81 & {$[.85, .88]$} \\
\hline (A3) Helpfulness & 1.65 & 1.38 & 1.67 & -2.47 & 6.04 & 8.52 & {$[.91, .93]$} \\
\hline (A4) Obligingness & 1.17 & 1.30 & 1.05 & -1.86 & 5.55 & 7.41 & {$[.84, .87]$} \\
\hline (A5) Modesty & .78 & 1.13 & .73 & -2.68 & 5.11 & 7.79 & {$[.82, .86]$} \\
\hline (A6) Good naturedness & 2.13 & 1.78 & 2.09 & -2.99 & 6.40 & 9.39 & {$[.74, .79]$} \\
\hline
\end{tabular}

Note. Descriptives of demographic and personality trait variables for the 624 participants (377 female, 243 male, 4 not disclosed). alphaCI = 1000 times iterated, 95\% bootstrapped percentile confidence intervals for Cronbach alpha coefficients of internal consistency. Cronbach alpha values were computed on the basis of 607 participants, due to some missing raw item values from study 1, see Stachl et al. (2017) for more details.

all performance metrics are reported in Table 4, plots for elastic net models are available in the project's repository (Stachl et al., 2019).

With the exception of the facet cheerfulness $\left(r_{\mathrm{md}}=.16, r_{\mathrm{sd}}\right.$ $=.12, R_{\mathrm{md}}^{2}=.00, R_{\mathrm{sd}}^{2}=.05, R M S E_{\mathrm{md}}=1.64, R M S E_{\mathrm{sd}}=$ $.13)$, the personality trait of extraversion and its facets were successfully predicted above baseline $\left(r_{\mathrm{md}}=.37, r_{\mathrm{sd}}=.09\right.$, $\left.R_{\mathrm{md}}^{2}=.11, R_{\mathrm{sd}}^{2}=.06, R M S E_{\mathrm{md}}=.70, R M S E_{\mathrm{sd}}=.06\right)$ on factor and facet levels. Most notably, the facet of sociableness was predicted with the highest $R^{2}$ of all criteria $\left(r_{\mathrm{md}}=.40\right.$, $r_{\mathrm{sd}}=.10, R_{\mathrm{md}}^{2}=.13, R_{\mathrm{sd}}^{2}=.07, R M S E_{\mathrm{md}}=1.60, R M S E_{\mathrm{sd}}=$ $.14)$. Besides sociableness, the facets friendliness $\left(r_{\mathrm{md}}=.24\right.$, 
Table 3

Pairwise Spearman correlations between big five personality traits and demographic variables.

\begin{tabular}{|c|c|c|c|c|c|c|c|}
\hline & gender & age & education & $\mathrm{O}$ & $\mathrm{C}$ & $\mathrm{E}$ & A \\
\hline age & $.10,[.02, .17]$ & & & & & & \\
\hline education & $.00,[-.08, .08]$ & $.33,[.25, .41]$ & & & & & \\
\hline $\mathrm{O}$ & $-.09,[-.17,-.02]$ & $.01,[-.08, .09]$ & $.08,[.00, .15]$ & & & & \\
\hline $\mathrm{C}$ & $-.06,[-.14, .02]$ & $.06,[-.03, .14]$ & $.05,[-.03, .13]$ & $.29,[.22, .36]$ & & & \\
\hline $\mathrm{E}$ & $-.09,[-.17,-.01]$ & $.00,[-.08, .07]$ & $-.04,[-.11, .03]$ & $.49,[.43, .55]$ & $.27,[.19, .34]$ & & \\
\hline A & $-.04,[-.12, .04]$ & $.08,[-.01, .15]$ & $-.02,[-.09, .05]$ & $.37,[.30, .44]$ & $.16,[.07, .23]$ & $.34,[.26, .40]$ & \\
\hline ES & $.27,[.19, .33]$ & $.03,[-.05, .11]$ & $-.05,[-.13, .03]$ & $.25,[.17, .32]$ & $.26,[.19, .33]$ & $.46,[.39, .52]$ & $.33,[.25, .40]$ \\
\hline
\end{tabular}

Note. Pairwise Spearman correlations between big five personality factors (measured with the BFSI), and demographic variables in the complete dataset. Gender was coded with $1=$ female and $2=$ male. Brackets contain $95 \%$ bootstrapped percentile confidence intervals with 1000 iterations.

$r_{\mathrm{sd}}=.09, R_{\mathrm{md}}^{2}=.04, R_{\mathrm{sd}}^{2}=.05, R M S E_{\mathrm{md}}=1.23, R M S E_{\mathrm{sd}}$ $=.11)$, assertiveness $\left(r_{\mathrm{md}}=.29, r_{\mathrm{sd}}=.11, R_{\mathrm{md}}^{2}=.06, R_{\mathrm{sd}}^{2}=\right.$ $\left..06, R M S E_{\mathrm{md}}=1.32, R M S E_{\mathrm{sd}}=.12\right)$, dynamism $\left(r_{\mathrm{md}}=.29\right.$, $r_{\mathrm{sd}}=.10, R_{\mathrm{md}}^{2}=.06, R_{\mathrm{sd}}^{2}=.06, R M S E_{\mathrm{md}}=1.52, R M S E_{\mathrm{sd}}$ $=.12)$, and adventurousness $\left(r_{\mathrm{md}}=.29, r_{\mathrm{sd}}=.11, R_{\mathrm{md}}^{2}=\right.$ $\left..06, R_{\mathrm{sd}}^{2}=.07, R M S E_{\mathrm{md}}=1.42, R M S E_{\mathrm{sd}}=.13\right)$ could be predicted above baseline. Behavioral patterns and semantic importance in Figure 2 suggest that often variables related to communication and social behavior, partially with regard to day and nighttime activity, were ranked as most important in the prediction models of extraversion and its facets. Furthermore, ALE-plots in Figure 3 indicate positive, non-linear relationships between various communication variables and dimensions of extraversion. Specifically, these plots show that for participants who on average made more outgoing calls per day and for those who on average called more different people per day, higher levels of extraversion were predicted on average. Similarly, higher daily mean numbers in the usage of WhatsApp and the mean daily number of outgoing calls led to higher on average predictions for sociableness. Furthermore, higher values for friendliness were predicted for participants with a higher standard deviation in the number of incoming calls per day and a lower irregularity of ringing events on weekends (IVI_call_ring_weekend). Relatively homogeneous patterns are visible for models across all facets of extraversion.

Openness and its facets were successfully predicted in our dataset $\left(r_{\mathrm{md}}=.29, r_{\mathrm{sd}}=.11, R_{\mathrm{md}}^{2}=.05, R_{\mathrm{sd}}^{2}=.05, R M S E_{\mathrm{md}}=\right.$ $\left..68, R M S E_{\mathrm{sd}}=.05\right)$. All facets of openness were also successfully predicted above baseline using random forest models. Of all openness-facets, openness to aesthetics showed the highest median prediction performance $\left(r_{\mathrm{md}}=.29, r_{\mathrm{sd}}=.12, R_{\mathrm{md}}^{2}=\right.$ $\left..05, R_{\mathrm{sd}}^{2}=.05, R M S E_{\mathrm{md}}=1.25, R M S E_{\mathrm{sd}}=.14\right)$, openness to imagination the lowest $\left(r_{\mathrm{md}}=.19, r_{\mathrm{sd}}=.13, R_{\mathrm{md}}^{2}=.01, R_{\mathrm{sd}}^{2}\right.$ $\left.=.05, R M S E_{\mathrm{md}}=1.35, R M S E_{\mathrm{sd}}=.10\right)$, with openness to feelings $\left(r_{\mathrm{md}}=.24, r_{\mathrm{sd}}=.09, R_{\mathrm{md}}^{2}=.03, R_{\mathrm{sd}}^{2}=.04, R M S E_{\mathrm{md}}\right.$ $\left.=2.03, R M S E_{\mathrm{sd}}=.16\right)$, openness to actions $\left(r_{\mathrm{md}}=.23, r_{\mathrm{sd}}=\right.$ $\left..11, R_{\mathrm{md}}^{2}=.03, R_{\mathrm{sd}}^{2}=.05, R M S E_{\mathrm{md}}=1.36, R M S E_{\mathrm{sd}}=.14\right)$ and openness to ideas ranging in between $\left(r_{\mathrm{md}}=.24, r_{\mathrm{sd}}=\right.$ $\left..11, R_{\mathrm{md}}^{2}=.04, R_{\mathrm{sd}}^{2}=.05, R M S E_{\mathrm{md}}=1.38, R M S E_{\mathrm{sd}}=.12\right)$. Unlike for extraversion, openness and its facets were best predicted by a diverse mix of behavioral variables. Specifically, the visual inspection of semantic importance indicators in Figure 2 suggests that app-usage, music listening behavior, and partially communication played particularly important roles. Specifically, ALE-plots in Figure 3 suggest non-linear predictor effects of music listening behavior in the respective models. For example, lower levels of openness were predicted for participants that listened to louder music on weekends and for participants with higher minimum values for music-energy and danceability. With the exception of openness to actions, similar and pronounced effects can be seen across the facets of openness. Besides music listening behavior, semantic importance measures show that app-usage and communication played an important role in the prediction of openness and its facets. Specifically, variables related to the usage of sportnews apps, the usage of the phone's camera, the length of text messages, and ringing events were identified as important across several facets. Whereas an increased usage of sportnews in general led to lower predicted values in openness dimensions, increased usage of the phone's camera and both higher and more varying length of text messages on average led to higher predicted values in those dimensions (see Figure 3 and the project's repository for ALE plots of all effects). Moreover, behavioral patterns in Figure 2 indicate differences in predictor-rankings across facets of openness - possibly hinting at the heterogeneity of this personality dimension (DeYoung, 2015; Mussel, Winter, Gelléri, \& Schuler, 2011). Whereas predictions in the model for the facet openness to actions have been mainly driven by communication related variables, models for openness to imagination and openness 
to aesthetics have mostly relied on variation in app usage and music preferences.

Conscientiousness was also successfully predicted above baseline $\left(r_{\mathrm{md}}=.31, r_{\mathrm{sd}}=.13, R_{\mathrm{md}}^{2}=.06, R_{\mathrm{sd}}^{2}=.05, R M S E_{\mathrm{md}}=\right.$ $\left..71, R M S E_{\mathrm{sd}}=.06\right)$. Additionally, all facets of conscientiousness were successfully predicted. In terms of prediction performance, the facet love of order ranked first $\left(r_{\mathrm{md}}=.31\right.$, $r_{\mathrm{sd}}=.13, R_{\mathrm{md}}^{2}=.07, R_{\mathrm{sd}}^{2}=.05, R M S E_{\mathrm{md}}=1.49, R M S E_{\mathrm{sd}}=$ $.12)$, followed by sense of duty $\left(r_{\mathrm{md}}=.29, r_{\mathrm{sd}}=.10, R_{\mathrm{md}}^{2}=\right.$ $\left..06, R_{\mathrm{sd}}^{2}=.04, R M S E_{\mathrm{md}}=1.34, R M S E_{\mathrm{sd}}=.11\right)$, ambition $\left(r_{\mathrm{md}}=.26, r_{\mathrm{sd}}=.12, R_{\mathrm{md}}^{2}=.04, R_{\mathrm{sd}}^{2}=.05, R M S E_{\mathrm{md}}=1.64\right.$, $\left.R M S E_{\mathrm{sd}}=.15\right)$, discipline $\left(r_{\mathrm{md}}=.22, r_{\mathrm{sd}}=.12, R_{\mathrm{md}}^{2}=.03\right.$, $\left.R_{\mathrm{sd}}^{2}=.05, R M S E_{\mathrm{md}}=1.43, R M S E_{\mathrm{sd}}=.15\right)$, caution $\left(r_{\mathrm{md}}\right.$ $=.20, r_{\mathrm{sd}}=.12, R_{\mathrm{md}}^{2}=.02, R_{\mathrm{sd}}^{2}=.05, R M S E_{\mathrm{md}}=1.31$, $\left.R M S E_{\mathrm{sd}}=.12\right)$, and competence $\left(r_{\mathrm{md}}=.19, r_{\mathrm{sd}}=.11, R_{\mathrm{md}}^{2}=\right.$ $\left..01, R_{\mathrm{sd}}^{2}=.05, R M S E_{\mathrm{md}}=1.19, R M S E_{\mathrm{sd}}=.13\right)$. Inspection of behavioral patterns and semantic importance indicators in Figure 2 suggest that variables related to general day and nighttime activity and app usage were especially important in the prediction models of conscientiousness and its facets. Predictor effects in Figure 3 suggest that higher levels of conscientiousness were predicted when the last traceable daily event on a participant's phone was on average detected before midnight. Relatedly, higher scores in conscientiousness were predicted for participants with temporal variation in the first daily event on average. Another important variable in the prediction model of conscientiousness was the daily variation in the standard deviation of accumulated inter-event time (sum of time between usage). Specifically, this effect suggests higher predicted scores in conscientiousness for lower scores in inter-event time variation. Similar and more pronounced patterns of predictor-influence can be seen across the majority of facets. As an exception, predictions in the model for the facet competence more heavily relied on mobility and communication-related variables. For example, predictor effects suggest higher predicted scores in competence with higher daily mean numbers of contacts and larger on average covered distances on weekends.

Furthermore, a number of additional predictor effects across dimensions of conscientiousness were discovered. In that regard, higher scores were in general predicted for higher mean usage numbers of weather apps, longer usage durations of a local public transportation app (de.swm.mvgfahrinfo.muenchen), longer and less variant usage times of the camera, and less variation in the usage of apps from the checkup and monitoring category. Unlike other facets of conscientiousness, predictions for the facet caution were lower if the cosine-similarity between contacted people on weekdays and on weekends was higher and if the mean valence of listened music on weekends was higher. Finally, for the facets love of order and sense of duty, a very specify behavior was found to be important the mean charge of the phone when it was disconnected from a charging cable. Consequent investigation of ALE-plots in Figure 3 suggest that higher average scores for both facets were predicted for phone disconnections at battery charges above $60 \%$.

For the personality dimension of emotional stability, only the facets of carefreeness $\left(r_{\mathrm{md}}=.22, r_{\mathrm{sd}}=.10, R_{\mathrm{md}}^{2}=.03, R_{\mathrm{sd}}^{2}\right.$ $\left.=.05, R M S E_{\mathrm{md}}=1.27, R M S E_{\mathrm{sd}}=.10\right)$, self-consciousness $\left(r_{\mathrm{md}}=.32, r_{\mathrm{sd}}=.09, R_{\mathrm{md}}^{2}=.08, R_{\mathrm{sd}}^{2}=.07, R M S E_{\mathrm{md}}=1.11\right.$, $\left.R M S E_{\mathrm{sd}}=.11\right)$, and self-control $\left(r_{\mathrm{md}}=.20, r_{\mathrm{sd}}=.11, R_{\mathrm{md}}^{2}\right.$ $\left.=.02, R_{\mathrm{sd}}^{2}=.05, R M S E_{\mathrm{md}}=.97, R M S E_{\mathrm{sd}}=.12\right)$ were predicted above baseline. Behavioral patterns in Figure 2 are rather distinct for the individual facets of emotional stability. Whereas variable importance measures for the model of self-consciousness suggest communication and social behavior variables as predictive, models for carefreeness and self-control suggest more diverse behavioral effects. The prediction of self-consciousness was most heavily influenced by calling behavior and the number of contacts per day. Specifically, positive - non-linear relationships of those variables on the predicted levels of self-consciousness were found (see Figure 3 and the project's repository, for visualizations of those effects). Predictions for the facet carefreeness were increased by various activities ranging from lower numbers in the daily mean number of screen unlock events, lower daily mean usage numbers of the Android email client, and lower variance in the usage of of strategy games. Besides other variables, predicted values in the dimension self-control were negatively influenced by higher entropy values in the usageduration of casual gaming apps and an increased variation in the usage duration of checkup and monitoring apps (e.g., Speedtest).

In the present analyses, the personality dimension of agreeableness could not be successfully predicted from the data, neither on factor nor on facet level $\left(r_{\mathrm{md}}=.05, r_{\mathrm{sd}}=.11, R_{\mathrm{md}}^{2}\right.$ $\left.=-.01, R_{\mathrm{sd}}^{2}=.05, R M S E_{\mathrm{md}}=.76, R M S E_{\mathrm{sd}}=.07\right)$.

\section{Discussion}

Results from the present study show how big five personality traits are manifested in patterns of smartphone-sensed behaviors. Furthermore, we demonstrate that these behavioral patterns can be used to automatically predict levels of individual personality traits. With modest prediction success, the trained models were able to predict the traits of extraversion, openness, conscientiousness, and single facets of emotional stability, above chance. Beyond the predictive performance, our results also provide insights into the types of behavior that were most relevant for the prediction of individual personality traits.

Our findings do not align well with previous results by Mønsted et al. (2018), who reported very limited success in the pre- 
Table 4

Median prediction performance per personality dimension and algorithm

\begin{tabular}{|c|c|c|c|c|c|c|c|}
\hline \multirow[b]{2}{*}{ Personality Dimension } & \multicolumn{3}{|c|}{ Random Forest } & \multicolumn{3}{|c|}{ Elastic Net } & \multirow{2}{*}{$\frac{\text { Baseline }}{\text { RMSE }}$} \\
\hline & $\mathrm{r}$ & $R^{2}$ & RMSE & $\mathrm{r}$ & $R^{2}$ & RMSE & \\
\hline (ES) Emotional Stability & .11 & .00 & .71 & .01 & -.01 & .71 & .71 \\
\hline (E) Extraversion & .37 & .11 & .70 & .26 & .05 & .72 & .74 \\
\hline (O) Openness & .29 & .05 & .68 & .25 & .04 & .69 & .71 \\
\hline (C) Conscientiousness & .31 & .06 & .71 & .16 & .00 & .73 & .74 \\
\hline (A) Agreeableness & .05 & -.01 & .76 & -.01 & -.01 & .75 & .75 \\
\hline (ES1) Carefreeness & .22 & .03 & 1.27 & .14 & .00 & 1.29 & 1.29 \\
\hline (ES2) Equanimity & .08 & -.01 & 1.07 & .02 & -.01 & 1.07 & 1.07 \\
\hline (ES3) Positive mood & .13 & .00 & 1.41 & -.05 & -.01 & 1.41 & 1.41 \\
\hline (ES4) Self consciousness & .32 & .08 & 1.11 & .25 & .04 & 1.14 & 1.17 \\
\hline (ES5) Self control & .20 & .02 & .97 & .10 & -.01 & 1.00 & 1.00 \\
\hline (ES6) Emotional robustness & .06 & -.02 & 1.18 & .01 & -.01 & 1.18 & 1.18 \\
\hline (E1) Friendliness & .24 & .04 & 1.23 & .18 & .01 & 1.26 & 1.28 \\
\hline (E2) Sociableness & .40 & .13 & 1.60 & .28 & .05 & 1.69 & 1.75 \\
\hline (E3) Assertiveness & .29 & .06 & 1.32 & .27 & .05 & 1.33 & 1.37 \\
\hline (E4) Dynamism & .29 & .06 & 1.52 & .24 & .03 & 1.56 & 1.58 \\
\hline (E5) Adventurousness & .29 & .06 & 1.42 & .26 & .04 & 1.45 & 1.48 \\
\hline (E6) Cheerfulness & .16 & .00 & 1.64 & .09 & -.01 & 1.66 & 1.67 \\
\hline (O1) Openness to imagination & .19 & .01 & 1.35 & .16 & .00 & 1.36 & 1.37 \\
\hline (O2) Openness to aesthetics & .29 & .05 & 1.25 & .28 & .06 & 1.25 & 1.29 \\
\hline (O3) Openness to feelings & .24 & .03 & 2.03 & .18 & .01 & 2.04 & 2.07 \\
\hline (O4) Openness to actions & .23 & .03 & 1.36 & .18 & .01 & 1.37 & 1.38 \\
\hline (O5) Openness to ideas & .24 & .04 & 1.38 & .13 & .00 & 1.41 & 1.42 \\
\hline (O6) Openness to value $\&$ norm & .22 & .02 & 1.00 & .17 & .01 & 1.01 & 1.02 \\
\hline (C1) Competence & .19 & .01 & 1.19 & .10 & -.01 & 1.21 & 1.21 \\
\hline (C2) Love of order & .31 & .07 & 1.49 & .22 & .03 & 1.51 & 1.55 \\
\hline (C3) Sense of duty & .29 & .06 & 1.34 & .16 & .01 & 1.38 & 1.40 \\
\hline (C4) Ambition & .26 & .04 & 1.64 & .20 & .02 & 1.66 & 1.68 \\
\hline (C5) Discipline & .22 & .03 & 1.43 & .11 & .00 & 1.47 & 1.47 \\
\hline (C6) Caution & .20 & .02 & 1.31 & .12 & .00 & 1.33 & 1.34 \\
\hline (A1) Willingness to trust & .05 & -.02 & 1.44 & .04 & -.01 & 1.42 & 1.43 \\
\hline (A2) Genuineness & .08 & -.02 & .95 & .00 & -.01 & .95 & .94 \\
\hline (A3) Helpfulness & .08 & -.01 & 1.39 & .09 & -.01 & 1.38 & 1.37 \\
\hline (A4) Obligingness & .09 & -.01 & 1.29 & .06 & -.02 & 1.30 & 1.29 \\
\hline (A5) Modesty & .05 & -.01 & 1.12 & .05 & -.01 & 1.12 & 1.12 \\
\hline (A6) Good naturedness & .03 & -.02 & 1.77 & -.04 & -.01 & 1.76 & 1.76 \\
\hline
\end{tabular}

Note. Median prediction metrics for each personality dimension and prediction algorithm, aggregated across all testsets in pearsons $r, R^{2}$, and RMSE. Perfomance metrics first computed for each cross-validation fold respectively and aggregated to the median. Bold coefficients: for $\mathrm{r}$ and $R^{2}$ where $R^{2}>0$ and RMSE is lower than baseline).

diction of big five personality traits from smartphone data. We achieved a similar prediction performance for the dimension of extraversion, but were able to predict scores for other big five dimensions above baseline. Although we used a sample of comparable size and similar demographic composition, our results suggest that the majority of big five personality traits indeed can be automatically predicted from a wide range of smartphone-sensed behaviors. Likely, this difference in pre- diction performance can be attributed to the type of data used in the present study. Whereas Mønsted et al. (2018) almost exclusively relied on data about communication behavior, we used a wide range of behavioral indicators for the prediction of personality traits. This is also in line with findings of Settanni et al. (2018), reporting increased predictability of personal traits through the combination of data-types. 


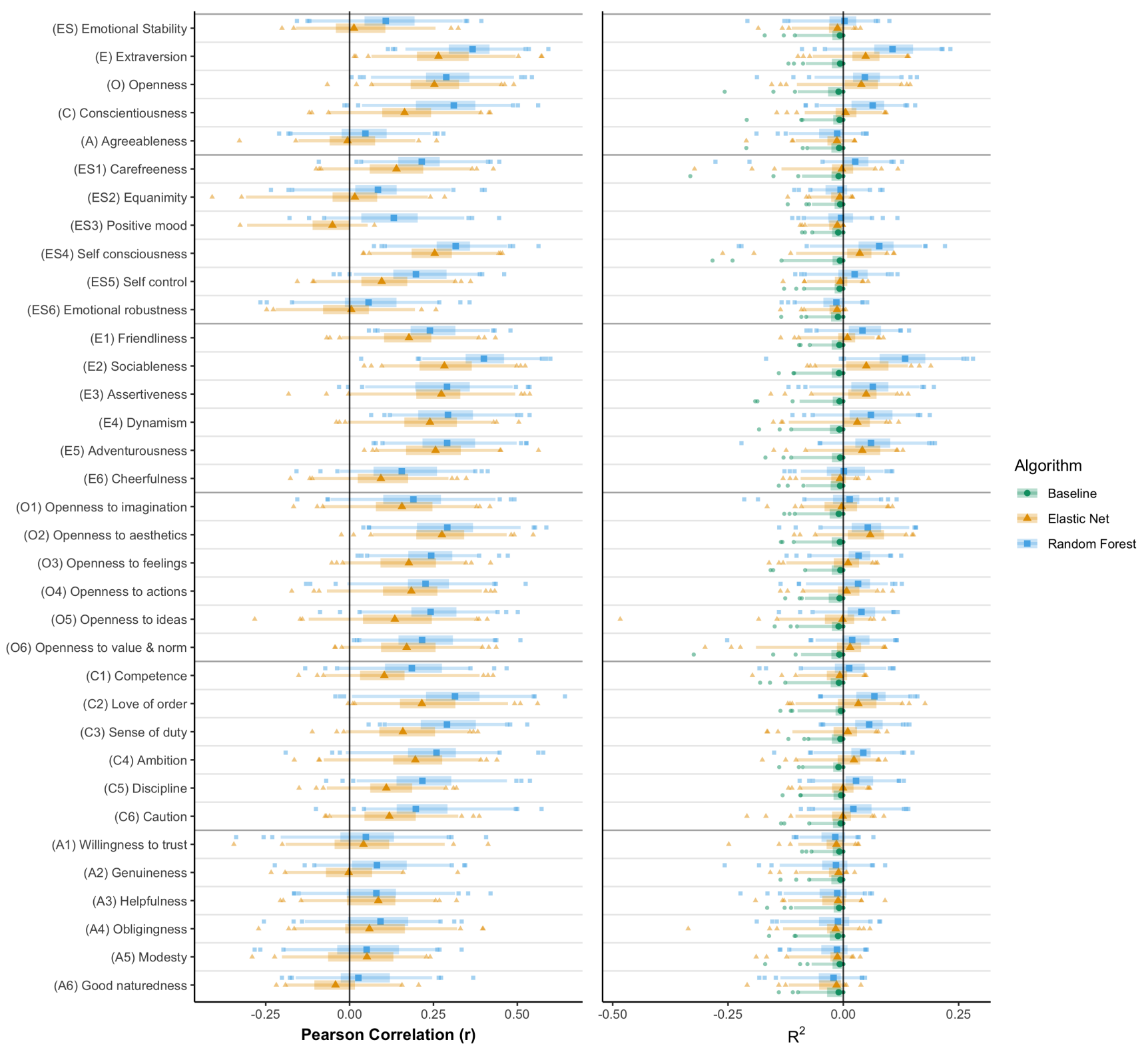

Figure 1. Box and whisker plot of prediction performance measures from repeated cross-validation for each personality factor and facet. The middle symbol represents the median, boxes indicate values between the $25 \%$ and $75 \%$ quantile, whiskers extend to the $2.5 \%$ and $97.5 \%$ quantiles. Outliers are indicated by single points. Figure available at https://osf.io/qnjdy/, under a CC-BY4.0 license.

Besides the prediction of big five personality trait scores from smartphone-sensed behaviors, our results shed more light on the behavioral underpinnings of personality per se. The investigation of the trained machine learning models reveals that various behavioral patterns played an important role in the prediction of individual personality traits. The personality dimension of extraversion describes an individual's affinity to show social, outgoing, assertive, and cheerful behaviors (Ashton, Lee, \& Paunonen, 2002; Goldberg, 1990). Relatedly, our results suggest that the prediction of extraversion was mainly driven by communication and social behavior (e.g., the mean number of outgoing calls per day, the irregularity of calls, or the mean number of WhatsApp uses per day). These findings are in line with previous findings - positively linking extraversion to the frequency and duration of communication behaviors (Harari et al., 2019; Mehl, Gosling, \& Pennebaker, 2006; Montag et al., 2014, 2015; Mønsted et al., 2018; Stachl et al., 2017). Furthermore, our analyses revealed that communication behavior at night was predictive for dimensions of extraversion (e.g., the mean number of calls per night). This finding also connects to previous literature reporting higher levels of extraversion for self-reported evening-type personalities (Adan et al., 2012; Randler et al., 2014). 

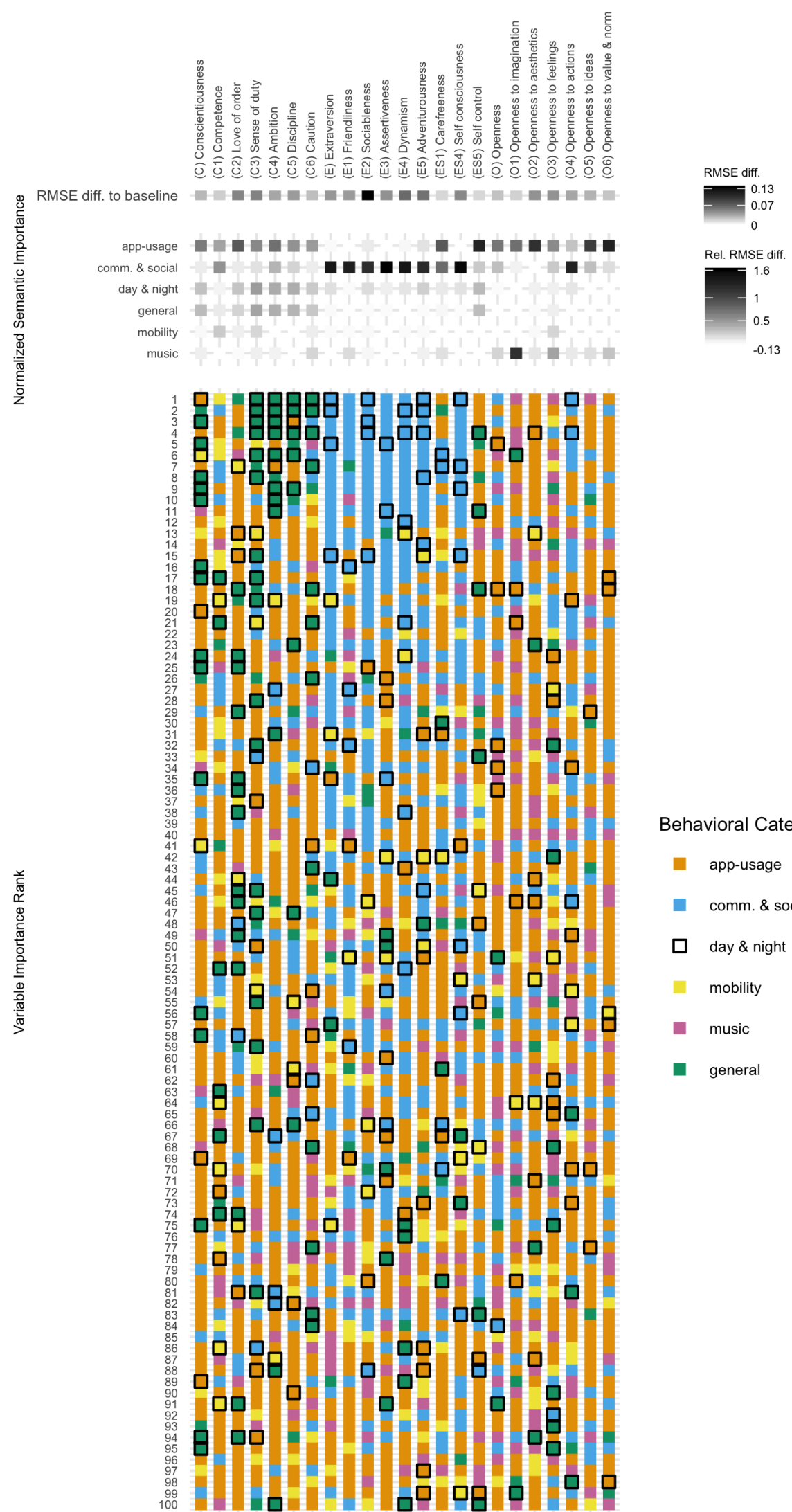

\section{Behavioral Category}

app-usage

comm. \& social

$\square$ day \& night

- mobility

music

- general

Figure 2. Visual indicators of overall model performance (top), semantic importance (middle) and variable importance patterns for random forest models, color-coded by behavior (bottom). Overall model performance: Grayscales represent the absolute difference in RMSE between a baseline model and the full prediction model, for each personality trait respectively. Normalized semantic importance: Grayscales represent the relative difference in RMSE between models including a semantic category and models with permuted values in the variables of the category (normalized at overall model performance). Behavioral Pattern plot: predictors with the highest variable importance in the respective model are at the top. Rows represent predictor ranks, columns represent individual models, squares represent variables, colors represent membership to semantic categories. Black frames indicate an additional day-night dependency of the respective variable. Figure available at https://osf.io/rt5cp/, under a CC-BY4.0 license 
(E) Extraversion

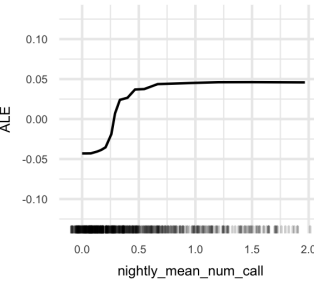

(E2) Sociableness

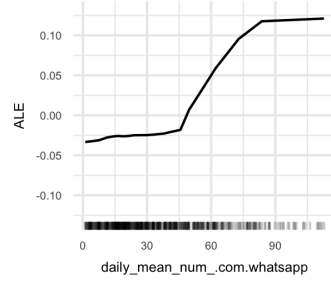

(O) Openness

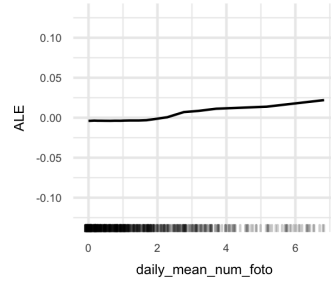

(O5) Openness to ideas

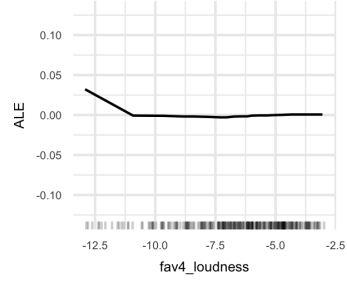

(C1) Competence

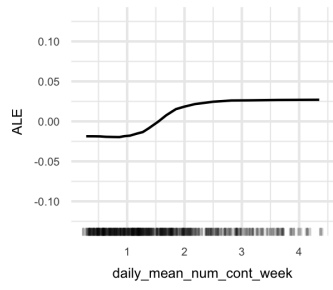

(C6) Caution

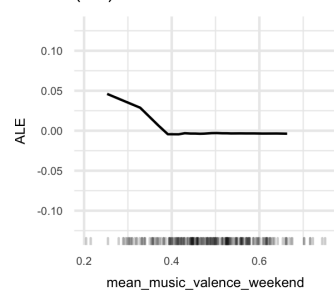

(E) Extraversion

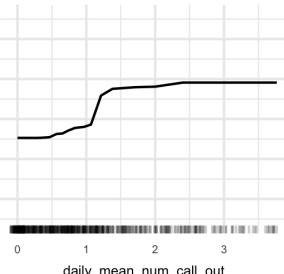

(E2) Sociableness

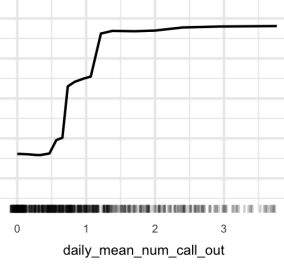

(O) Openness

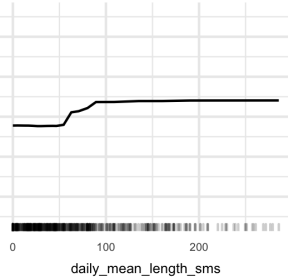

(C) Conscientiousness

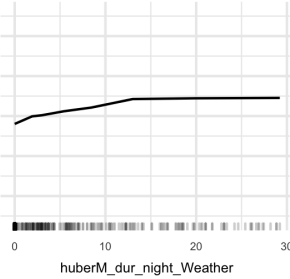

(C3) Sense of duty

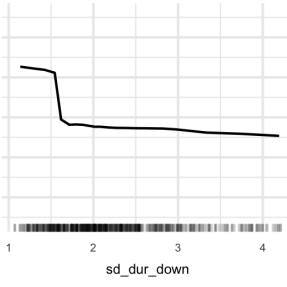

(ES1) Carefreeness

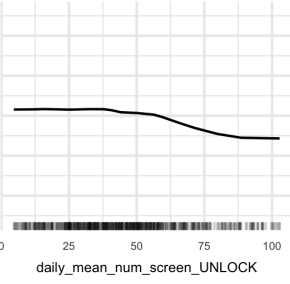

(E) Extraversion

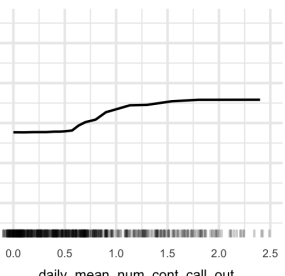

(O) Openness

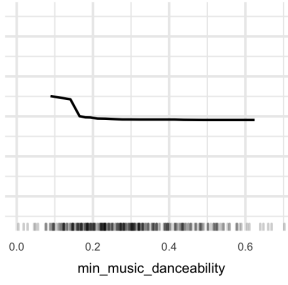

(O1) Openness to imagination

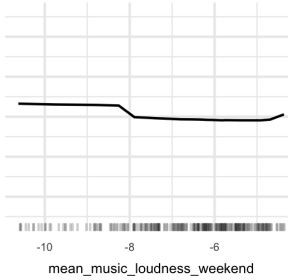

(C) Conscientiousness

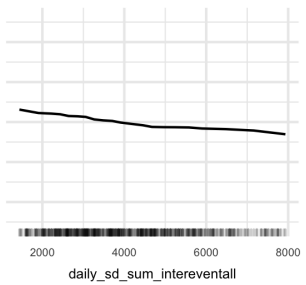

(C3) Sense of duty

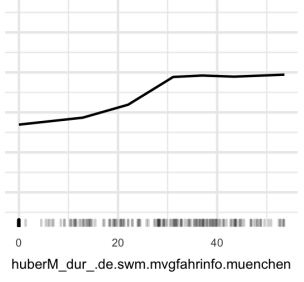

(ES4) Self consciousness

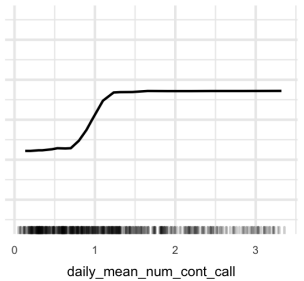

(E1) Friendliness

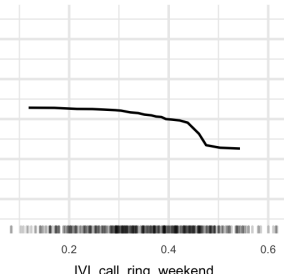

(O) Openness

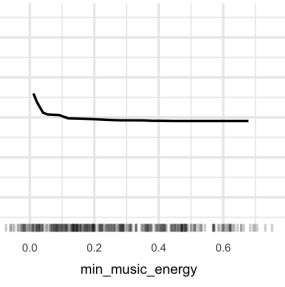

(O3) Openness to feelings

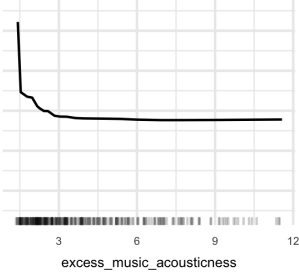

(C) Conscientiousness

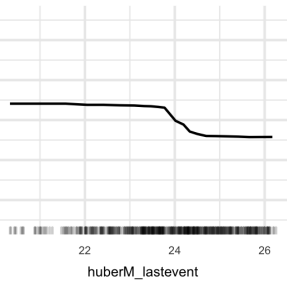

(C2) Love of order

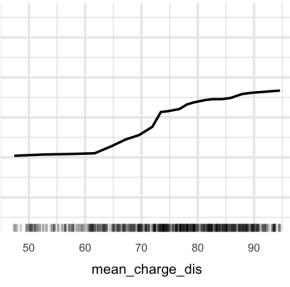

(ES5) Self control

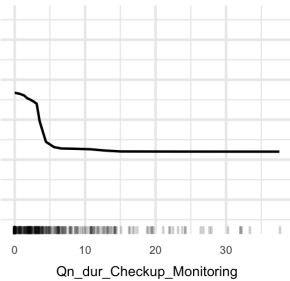

(E1) Friendliness

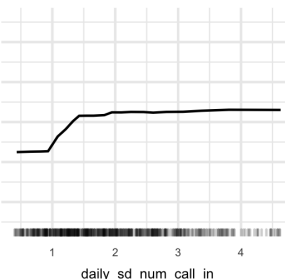

(O) Openness

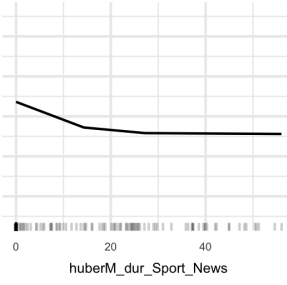

(O4) Openness to actions

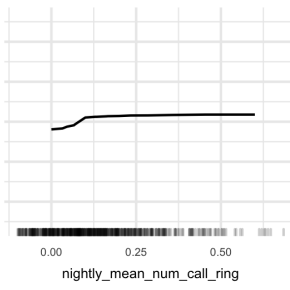

(C1) Competence

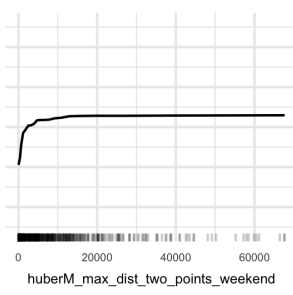

(C6) Caution

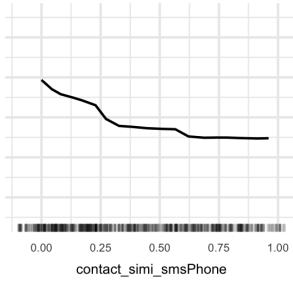

(ES5) Self control

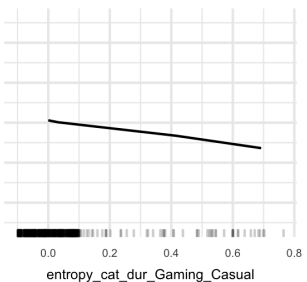

Figure 3. Selected accumulated local effect plots. Plots indicate how predictions in a given model changed on average with regard to different values in local value-areas of the respective predictor variable. ALE values are centered around zero. Figure available at https://osf.io/rtf7e/, under a CC-BY4.0 license. 
The personality dimension of openness is described as peoples' affinity to new experiences, values, ideas, actions, feelings, and aesthetics (DeYoung, 2015). In the present study, openness was successfully predicted, across all facets. The inspection of behavioral patterns in variable importance suggested a diverse mix of behaviors that played a role in the prediction models for openness and its facets. However, preferences in music consumption and app usage seem to have been particularly important for the prediction of openness dimensions. Specifically, our results suggest that participants with higher predicted values on openness dimensions preferred more quiet, less energetic, and less danceable songs. Although we used slightly different metrics, our results correspond with previous findings that reported preferences for more complex, sophisticated and presumably less danceable music for people with higher scores in openness (Dunn et al., 2012; Greenberg et al., 2016; Langmeyer et al., 2012). Beyond the link between music consumption and openness, the importance of camera usage and photography could hint at the desire to capture and share novel stimuli and at an affinity for aesthetics (Hunt \& Langstedt, 2014). Similar to findings in our study, links between the length of communication events (text messages and word length) for people with higher scores in openness were also found in past research (Harari et al., 2019; Mehl et al., 2006; Yarkoni, 2010). This finding could indicate the tendency of open-minded people to prefer novelty, originality, and variety in their behavior and experiences. Furthermore, Yarkoni (2010) argued that due to a positive link between levels of openness and cognitive abilities, highly open individuals might more often use longer and more complex words (Gignac, Stough, \& Loukomitis, 2004). Therefore, a more complex style of expression could have led to longer on average text messages. Although the negative effect of sport-news apps usage on predicted levels of openness could hint at a similar mechanism, we refrain from any further interpretation as no previous effects have been reported. In general, one can only speculate about the majority of these findings, since little previous work exists most variables interact with many others. All found predictor effects should therefore be adequately replicated in prospective, confirmatory research.

The trait of conscientiousness has been defined as individual differences in goal-directed and self-disciplined behavior, the engagement in planning behavior, and the obedience to norms and rules (Roberts, Bogg, Walton, Chernyshenko, \& Stark, 2004). Therefore, conscientiousness is considered one of the most relevant personality dispositions for practical applications and the prediction of important life outcomes (e.g., hiring decisions and life satisfaction, Ozer \& Benet-Martínez, 2006; Roberts et al., 2007). In this study, individual levels of conscientiousness and its facets were predicted above baseline. Directly related to the definition of conscientiousness, behaviors that signify when participants used their telephone for the first and last time per day, and variables related to the regularity of their usage patterns, were important in the models. These behavioral patterns are in line with past research related to how conscientious people report to behave (Jackson et al., 2010). The importance of behavioral routine for the prediction of conscientiousness levels in the present study aligns well with findings by Jackson et al. (2010), who reported one of the highest, negative associations between self-reported behaviors and self-reported levels of conscientiousness for the item "Break daily routine". Furthermore, Jackson et al. (2010) reported multiple additional associations between levels of conscientiousness, daily routines, and planning behavior (e.g., "Miss the bus", "Get to work on time", and "Leave for work at the exact time I had planned"). Those associations line up with the importance of increased public transportation app usage, weather app usage and increased battery charges in our prediction models: highly conscientious individuals might more often check the weather forecast and public transportation schedules to be prepared for daily eventualities. Moreover, earlier on-average first and last events led to higher predicted values on conscientiousness and its facets. This finding likely relates to previous studies that associated conscientiousness with a preference for earlier wake-up times (Tsaousis, 2010). A preference for morning activity has in turn has been positively associated with the self-reported regularity of sleeping behaviors during the week versus on weekends in past research (Roenneberg, Wirz-Justice, \& Merrow, 2003).

Individual differences in emotional stability relate to peoples' propensity to experience negative emotions, to perceive situations as stressful, and the ability to control urges (Whiteside \& Lynam, 2001). In our study, the emotional stability facets of self-consciousness, carefreeness, and self-control were predicted above chance. Low levels of emotional stability have repeatedly been linked to negative life outcomes such as low happiness, anxiety, depression, unsatisfying relationships, and even psychopathology (Ormel et al., 2013; Ozer \& BenetMartínez, 2006). Those in turn have been associated with decreased (social) activity and reduced mobility (Burton et al., 2013; Saeb et al., 2016). Presently discovered behavioral patterns of increased direct communication frequency and larger numbers of called contacts for higher values in selfconsciousness (e.g., the mean number of contacts called per day) point in a similar direction. Predictions for the facet carefreeness were driven by how often people had unlocked their phones on average, the loudness and valence of most listened songs, the number of different songs per day, and the usage of strategy games. Emotional stability has been linked to preferences in music listening behaviors on both categorical- and genre-level (Dunn et al., 2012; Greenberg et al., 2016). The importance of musical variables in this prediction model could highlight the affective component of this personality dimension. However, ultimately the complex 
interaction of these distinct behaviors prevents any further conclusions. A slightly clearer picture emerges with regard to the dimension of self-control. In general, behavioral patterns suggest that higher scores in self-control were predicted for participants with a better ability to avoid distractions by their phones (e.g., by playing games, making calls, or opening apps). Furthermore higher event-irregularity led to lower predictions in self-control. The latter of these behavioral patterns intuitively lines up with the definition of that personality dimension - the ability to control urges.

\section{Outlook \& Limitations}

Although the majority of big five personality trait dimensions could be predicted above chance, the median prediction performance in 12 out of 35 dimensions did not exceed the baseline. Precisely, all dimensions of agreeableness, three facets of emotional stability, and the extraversion-facet cheerfulness were not predicted successfully. Although it remains unclear why these dimensions could not be predicted above baseline, a number of possible explanations comes to mind. Agreeableness is a personality dimension that describes how cooperative, trustful, and respectful people are of others (Graziano \& Tobin, 2009). The inability to predict agreeableness and its facets is surprising as some previous studies have reported successful predictions from both social media data (Youyou et al., 2015) and text data (Park et al., 2015). However, agreeableness is a personality dimension that might rather be related to the quality of behavior and somewhat less related to the quantity of behavior. For example, both an agreeable and a disagreeable person might engage in a similar amount of communication events. However, an agreeable person will likely communicate kinder and show less offensive language (Park et al., 2015). Considering the scope of the data in this study, it is likely that not enough information about the quality aspects of behavior was available. Similarly, prediction efforts for emotional stability facets of equanimity, positive mood, and emotional robustness as well as the extraversion facet of cheerfulness might have failed due to the fact that not enough data about personal affect and behavior in reaction to the behavior of others (e.g., staying calm in stressful situations) was available in the dataset. Solely the behavioral category of music consumption includes behaviors that are associated with personal mood and affect (Millgram, Joormann, Huppert, \& Tamir, 2015). However, previous results also suggest that listening to music is used for mood-regulation purposes (Saarikallio \& Erkkilä, 2007; Skånland, 2013; Thoma, Ryf, Mohiyeddini, Ehlert, \& Nater, 2012). Under this assumption, music consumption would help to control and stabilize emotions (e.g., listening to happy music when sad). Therefore, the actual direction of this effect is unknown and both directions of action are plausible. Although single music variables were important for the prediction of facets of emotional stability (e.g., carefreeness), more research using objective data about music consumption is needed.

Despite the wide range of behaviors that were available in the present study, the modest prediction performances of our results indicate that we could not capture the full spectrum of personality-relevant behaviors with our dataset. Prospective studies should aim to include additional behaviors that can be collected with smartphone-sensing methods. Those could include data from social networks (Settanni et al., 2018), audio-data from on-board microphones (Mohammadi \& Vinciarelli, 2012), individual language use from texts (Park et al., 2015), visited websites (Kosinski, Bachrach, Kohli, Stillwell, \& Graepel, 2014), the content of photos (Ferwerda, Schedl, $\&$ Tkalcic, 2016), data about physical activity (Lathia et al., 2017), and more. However, as the additional collection of these data would drastically increase the sensitivity of the dataset and would therefore require much more advanced measures of data protection, we chose not to include these in the past studies.

With regard to the missing predictability of agreeableness, prospective research should additionally focus on the content and the quality of behavior. This could be achieved through sentiment analyses in texts and through the recognition of more complex behaviors (e.g., sensing anger in conversations). Furthermore, although the datasets used in this study were very similar, differences in study procedures could have had influences on participants' behaviors. Also, the datasets were collected over the course of four years with different versions of the PhoneStudy app. This might have introduced some systematic differences between parameters in the dataset (e.g., changes in the popularity of apps, changes in Android libraries - needed for logging). Although we addressed this issue through the semantic categorization of apps and intensive pre-testing before each study, prediction performance could possibly be increased further with a more coherent dataset.

\section{Implications}

The results of the present study hold implications for researchers and society alike. Primarily, our results show that distinct patterns in smartphone usage can be indicative of individual personality trait levels. As most commercial apps from big companies are enabled to collect these parameters in the course of naturalistic user interaction, possible inferences that can be made from these data should be made transparent to users. Precisely, as suggested by our results, the usage of specific applications and services could reveal various aspects of individual personality traits. For example (and in oversimplified terms), while basic measures of behavioral regularity could provide inferences about levels of conscientiousness, music consumption could be informative about levels of openness, and communication data could indicate individual 
extraversion levels. Whereas the approximate prediction of extraversion could be used to tailor marketing campaigns to individuals (Matz et al., 2017), the approximate prediction of openness could be used to provide more susceptible political campaign messages to voters (Roets, Cornelis, \& Van Hiel, 2014). Although both predictions could influence the behavior of individuals, effects of varying significance are conceivable.

It has to be noted that the reported prediction performances by far do not reach the precision of a personality profile obtained from a state-of-the art personality inventory. Nevertheless, despite the fact that the reported results were obtained from a rather small sample of 624 participants, prediction performances in the present study are comparable to those found in previous studies of social media usage (Settanni et al., 2018), partially using much larger samples (Kosinski et al., 2013). Furthermore, any commercial collector of phone usage data would have access to much larger quantities of user data, potentially allowing for higher predictive performance with more usage-parameters. Most importantly, the abuse of user data will likely not require prediction performance in the range of state-of-the art personality inventories. As previous research has demonstrated, even the use of rough personality judgments can have a non-negligible impact on behavior (Matz et al., 2017). Although we have no ready solution to this problem, our findings should stimulate a debate about how individual privacy rights can be protected knowing that even basic phone usage parameters can allow inferences about individual personality traits.

In addition to implications on individual privacy, the present work reports on associations and cross-validated effects of behavior and personality traits that have never been reported before. We hypothesize that along with the continuously increasing availability of digital indicators of personality in traceable behaviors, prediction performances for personality traits will gradually increase. However, prediction performances will eventually peak at an upper limit: the perfect prediction of answers in a personality trait questionnaire. Due to a myriad of almost uncontrollable influences (P. M. Podsakoff, MacKenzie, \& Podsakoff, 2012; P. M. Podsakoff, MacKenzie, Lee, \& Podsakoff, 2003; Vaerenbergh \& Thomas, 2013), self-reports of personality are likely not the optimum way of measuring personality differences in the first place.

This is the case although some questionnaires already use complex underlying measurement models (e.g., the BFSI in the present study) that aim to measure scores on the presumably underlying latent variable instead of items responses. As personality traits are primarily used to predict life-outcomes (e.g., relationship satisfaction, work performance; Ozer \& Benet-Martínez, 2006; Roberts et al., 2007), this raises the question of why not to directly utilize digital behavioral indicators as predictors of those. We see at least two problems with this approach. First, many digital indicators are unstable and could lose their relevance to personality traits over time (e.g., popular apps, technological developments). Thus, we anticipate that prospective studies will focus on the identification of relatively basic and stable dimensions of individual differences that can be quantified in traceable parameters. Secondly, we believe that it will be of the utmost importance to develop metrics that are understandable to people. In this study we undertook first steps toward this goal by categorizing variables in semantic categories. However, ideally this step could be automated with more data-driven structure-finding methodologies (e.g., cluster analyses, deep-learning). This will help to expand our knowledge of systematic differences in human personality and support the validation of predictive models.

Nevertheless, we do propose to increase the focus on the prediction of relevant life outcomes and prospective behavior. Specifically, researchers should contrast the prediction capabilities of digital footprints, self-reported personality scores and the combination of both. This could advance the debate about the optimum level of granularity at which personality traits should be conceptualized at (Ashton, Paunonen, \& Lee, 2014). So far, five to six dimensions (Ashton \& Lee, 2007; Goldberg, 1990; McCrae \& John, 1992) have mostly been deemed as sufficient. However, personality might also be defined as a high-dimensional representation of all possible combinations in traceable behavior, feelings, situations, and experience.

\section{Conclusion}

This work shows that personality trait levels can be predicted above chance from behavior, collected from off-the-shelf smartphones. While most previous work has focused on single, isolated types of behaviors, in this study we demonstrated that distinct patterns of semantically different behaviors are predictive for individual levels of big five personality traits. First, our findings suggest that everyday behavior on smartphones can allow for inferences on individual personality trait levels. Second, our findings suggest that research in behavioral sciences and personality psychology in particular could benefit from the inclusion of diverse behavioral indicators. Finally, the results of the present study highlight that smartphone-sensing methods enable the comprehensive and ecologically valid data collection of semantically different behaviors in the wild.

\section{Acknowledgements}

We thank everybody who contributed to the creation of this article. Florian Pargent who provided valuable feedback on earlier versions of this manuscript. The students that helped with recruitment, programming, testing, and many other tasks: 
Larissa Nina Nadine Sust, Florian Bemmann, Marius Schiele, Alexandra Dietl, Victoria Gebhardt, Sarah Huber, Martin Fröhlich, Miriam Metz, Florian Lehmann, Phuong-Anh Vu, Daniela Becker, Jakob Kaiser, Peter Ehrich and Matthias Lamm. We also thank the Schuhfried $\mathrm{GmbH}$ for providing the BFSI materials for implementation in our software.

\section{References}

Adan, A., Archer, S. N., Hidalgo, M. P., Di Milia, L., Natale, V., \& Randler, C. (2012). Circadian Typology: A Comprehensive Review. Chronobiology International, 29(9), 1153-1175. doi:10.3109/07420528.2012.719971

Anderson, M., \& Jiang, J. (2018). Teens, Social Media and Technology 2018. Pew Research Center. Retrieved from http://www.pewinternet.org/2018/05/ 31/teens-social-media-technology-2018/

Apley, D. W. (2016). Visualizing the Effects of Predictor Variables in Black Box Supervised Learning Models. Retrieved from http://arxiv.org/abs/1612.08468

Arendasy, M. (2009). BFSI: Big-Five Struktur-Inventar (Test \& Manual). Mödling: SCHUHFRIED GmbH.

Ashton, M. C., \& Lee, K. (2007). Empirical, Theoretical, and Practical Advantages of the HEXACO Model of Personality Structure. Personality and Social Psychology Review, 11(2), 150-166. doi:10.1177/1088868306294907

Ashton, M. C., Lee, K., \& Paunonen, S. V. (2002). What is the central feature of extraversion? Social attention versus reward sensitivity. Journal of Personality and Social Psychology, 83(1), 245-252. doi:10.1037/00223514.83.1.245

Ashton, M. C., Paunonen, S. V., \& Lee, K. (2014). On the validity of narrow and broad personality traits: A response to Salgado, Moscoso, and Berges (2013). Personality and Individual Differences, 56, 24-28. doi:10.1016/j.paid.2013.08.019

Au, Q. (2019). Fxtract: Feature extraction from grouped data. Retrieved from https://CRAN.R-project.org/ package $=$ fxtract

Azucar, D., Marengo, D., \& Settanni, M. (2018). Predicting the Big 5 personality traits from digital footprints on social media: A meta-analysis. Personality and Individual Differences, 124, 150-159. doi:10.1016/j.paid.2017.12.018

Barrick, M. R., \& Mount, M. K. (1991). The Big Five Personality Dimensions and Job Performance: A Meta-Analysis. Personnel Psychology, 44(1), 1-26. doi:10.1111/j.1744-6570.1991.tb00688.x

Baumeister, R. F., Vohs, K. D., \& Funder, D. C. (2007). Psychology as the Science of Self-Reports and Finger Movements: Whatever Happened to Actual Behavior? Perspectives on Psychological Science, 2(4), 
396-403. doi:10.1111/j.1745-6916.2007.00051.x

Binder, M. (2018). MlrCPO: Composable preprocessing operators and pipelines for machine learning. Retrieved from https://CRAN.R-project.org $/$ package $=\mathrm{mlrCPO}$

Bischl, B., \& Lang, M. (2015). ParallelMap: Unified interface to parallelization back-ends. Retrieved from https://CRAN.R-project.org/package=parallelMap

Bischl, B., Lang, M., Kotthoff, L., Schiffner, J., Richter, J., Studerus, E., ... Jones, Z. M. (2016). mlr: Machine Learning in R. Journal of Machine Learning Research, 17, 1-5. Retrieved from https://github. com/mlr-org/mlr

Bischl, B., Mersmann, O., Trautmann, H., \& Weihs, C. (2012). Resampling Methods for Meta-Model Validation with Recommendations for Evolutionary Computation. Evolutionary Computation, 20(2), 249-275. doi:10.1162/EVCO_a_00069

Breiman, L. (2001). Random Forests. Machine Learning, 45(1), 5-32. doi:10.1023/A:1010933404324

Burton, C., McKinstry, B., Szentagotai Tătar, A., SerranoBlanco, A., Pagliari, C., \& Wolters, M. (2013). Activity monitoring in patients with depression: A systematic review. Journal of Affective Disorders, 145(1), 21-28. doi:10.1016/j.jad.2012.07.001

Casalicchio, G., Molnar, C., \& Bischl, B. (2019). Visualizing the feature importance for black box models. In Energy transfer processes in polynuclear lanthanide complexes (pp. 655-670). Springer Singapore. doi:10.1007/978-3-030-10925-7_40

Ching, C. M., Church, A. T., Katigbak, M. S., Reyes, J. A. S., Tanaka-Matsumi, J., Takaoka, S., ... Ortiz, F. A. (2014). The manifestation of traits in everyday behavior and affect: A five-culture study. Journal of Research in Personality, 48, 116. doi:10.1016/j.jrp.2013.10.002

Chittaranjan, G., Blom, J., \& Gatica-Perez, D. (2013). Mining large-scale smartphone data for personality studies. Personal and Ubiquitous Computing, 17(3), 433450. doi:10.1007/s00779-011-0490-1

Costa, P. T., \& McCrae, R. R. (1980). Influence of extraversion and neuroticism on subjective wellbeing: Happy and unhappy people. Journal of Personality and Social Psychology, 38(4), 668-678. doi:10.1037/0022-3514.38.4.668

Costa, P. T., \& Widiger, T. A. (Eds.). (1994). Personality Disorders and the Five-Factor Model of Personality. Washington: American Psychological Association. doi:10.1037/10140-000

Costa, P., Jr, Terracciano, A., \& McCrae, R. R. (2001). Gender Differences in Personality Traits across Cultures: Robust and surprising Findings. Journal of Personality and Social Psychology, 81(2), 322-331. doi:10.1037/0022-3514.81.2.322

De Montjoye, Y.-A., Quoidbach, J., Robic, F., \& Pentland, A. (2013). Predicting personality using novel mobile phone-based metrics. In Proceedings of the 6th International Conference on Social Computing, Behavioral-Cultural Modeling and Prediction (pp. 48-55). Berlin, Heidelberg: Springer-Verlag. doi:10.1007/978-3-642-37210-0_6

De Raad, B. (2000). The Big Five Personality Factors: The psycholexical approach to personality. Hogrefe \& Huber Publishers.

DeYoung, C. G. (2015). Openness/intellect: A dimension of personality reflecting cognitive exploration. In M. Mikulincer, P. R. Shaver, M. L. Cooper, \& R. J. Larsen (Eds.), APA handbook of personality and social psychology, volume 4: Personality processes and individual differences (pp. 369-399). Washington, DC, US: American Psychological Association. doi:10.1037/14343-017

Duggan, K. A., Friedman, H. S., McDevitt, E. A., \& Mednick, S. C. (2014). Personality and healthy sleep: the importance of conscientiousness and neuroticism. PloS One, 9(3), e90628. doi:10.1371/journal.pone.0090628

Dunn, P. G., Ruyter, B. de, \& Bouwhuis, D. G. (2012). Toward a better understanding of the relation between music preference, listening behavior, and personality. Psychology of Music, 40(4), 411-428. doi:10.1177/0305735610388897

Ellis, D. A. (2019). Are smartphones really that bad? Improving the psychological measurement of technologyrelated behaviors. Computers in Human Behavior, 97, 60-66. doi:10.1016/J.CHB.2019.03.006

Ferwerda, B., Schedl, M., \& Tkalcic, M. (2016). Using Instagram Picture Features to Predict Users' Personality. In Q. Tian, N. Sebe, G.-J. Qi, B. Huet, R. Hong, \& X. Liu (Eds.), MultiMedia modeling, mmm 2016 (Vol. 9516, pp. 850-861). Springer International Publishing. doi:10.1007/978-3-319-27671-7_71

Fisher, A., Rudin, C., \& Dominici, F. (2018). Model Class Reliance: Variable Importance Measures for any Machine Learning Model Class, from the "Rashomon" Perspective. Retrieved from http://arxiv.org/abs/ 


\subsection{9}

Friedman, J., Hastie, T., \& Tibshirani, R. (2010). Regularization Paths for Generalized Linear Models via Coordinate Descent. Journal of Statistical Software, 33(1), 1-22. doi:10.18637/jss.v033.i01

Funder, D. C. (2001). Personality. Annual Review of Psychology, 52(1), 197-221. doi:10.1146/annurev.psych.52.1.197

Furr, R. M. (2009). Personality psychology as a truly behavioural science. European Journal of Personality, 23(5), 369-401. doi:10.1002/per.724

Gignac, G. E., Stough, C., \& Loukomitis, S. (2004). Openness, intelligence, and self-report intelligence. Intelligence, 32(2), 133-143. doi:10.1016/J.INTELL.2003.10.005

Gladstone, J. J., Matz, S. C., \& Lemaire, A. (2019). Can Psychological Traits Be Inferred From Spending? Evidence From Transaction Data. Psychological Science, 095679761984943. doi:10.1177/0956797619849435

Goldberg, L. R. (1990). An Alternative "Description of Personality": the Big-Five Factor Structure. Journal of Personality and Social Psychology, 59(6), 1216-29. doi:10.1037//0022-3514.59.6.1216

Google. (2018). Google Elevation API. Retrieved from https:/developers.google.com/maps/ documentation/elevation/intro

Google. (2019). Android Apps on Google Play. Retrieved from https://play.google.com/store/apps

Gosling, S. D., John, O. P., Craik, K. H., \& Robins, R. W. (1998). Do people know how they behave? Selfreported act frequencies compared with on-line codings by observers. Journal of Personality and Social Psychology, 74(5), 1337-1349. doi:10.1037/00223514.74.5.1337

Graziano, W. G., \& Tobin, R. M. (2009). Agreeableness. In M. R. Hoyle \& L. R. H (Eds.), Handbook of individual differences in social behavior (pp. 46-61). New York, NY, US: Guilford Press.

Greenberg, D. M., Kosinski, M., Stillwell, D. J., Monteiro, B. L., Levitin, D. J., \& Rentfrow, P. J. (2016). The Song Is You: Preferences for Musical Attribute Dimensions Reflect Personality. Social Psychological and Personality Science, 7(6), 597-605. doi:10.1177/1948550616641473

Gregorutti, B., Michel, B., \& Saint-Pierre, P. (2015). Grouped variable importance with random forests and appli- cation to multiple functional data analysis. Computational Statistics $\mathcal{E}$ Data Analysis, 90, 15-35. doi:https://doi.org/10.1016/j.csda.2015.04.002

Gregory, R. J. (2013). The History of Psychological Testing. In Psychological testing: History, principles, and applications. (7th ed., pp. 32-58). Pearson.

Guidotti, R., Monreale, A., Ruggieri, S., Turini, F., Pedreschi, D., \& Giannotti, F. (2018). A Survey Of Methods For Explaining Black Box Models. Retrieved from https://arxiv.org/abs/1802.01933

Harari, G. M., Gosling, S. D., Wang, R., \& Campbell, A. T. (2015). Capturing Situational Information with Smartphones and Mobile Sensing Methods. European Journal of Personality, 29(5), 509-511. doi:10.1002/per.2032

Harari, G. M., Lane, N. D., Wang, R., Crosier, B. S., Campbell, A. T., \& Gosling, S. D. (2016). Using Smartphones to Collect Behavioral Data in Psychological Science: Opportunities, Practical Considerations, and Challenges. Perspectives on Psychological Science, 11(6), 838-854. doi:10.1177/1745691616650285

Harari, G. M., Müller, S. R., Stachl, C., Wang, R., Wang, W., Bühner, M., ... Gosling, S. D. (2019). Sensing sociability: Individual differences in young adults' conversation, calling, texting, and app use behaviors in daily life. Journal of Personality and Social Psychology. doi:10.1037/pspp0000245

Huber, P. J. (1981). Robust Statistics. Hoboken, NJ, USA: John Wiley \& Sons, Inc. doi:10.1002/0471725250

Hunt, D. S., \& Langstedt, E. (2014). The Influence of Personality Factors and Motives on Photographic Communication. The Journal of Social Media in Society, 3(2). Retrieved from http://thejsms.org/index.php/ TSMRI/article/view/68

Jackson, J. J., Wood, D., Bogg, T., Walton, K. E., Harms, P. D., \& Roberts, B. W. (2010). What do conscientious people do? Development and validation of the Behavioral Indicators of Conscientiousness (BIC). Journal of Research in Personality, 44(4), 501-511. doi:10.1016/j.jrp.2010.06.005

Jed Wing, M. K. C. from, Weston, S., Williams, A., Keefer, C., Engelhardt, A., Cooper, T., ... Hunt., T. (2018). Caret: Classification and regression training. $\mathrm{Re}-$ trieved from https://CRAN.R-project.org/package= caret

Kim, Y., Briley, D. A., \& Ocepek, M. G. (2015). Differential innovation of smartphone and applica- 
tion use by sociodemographics and personality. Computers in Human Behavior, 44, 141-147. doi:10.1016/j.chb.2014.11.059

Kosinski, M., Bachrach, Y., Kohli, P., Stillwell, D., \& Graepel, T. (2014). Manifestations of user personality in website choice and behaviour on online social networks. Machine Learning, 95(3), 357-380. doi:10.1007/s10994-013-5415-y

Kosinski, M., Stillwell, D., \& Graepel, T. (2013). Private traits and attributes are predictable from digital records of human behavior. Proceedings of the National Academy of Sciences of the United States of America, 110(15), 5802-5. doi:10.1073/pnas.1218772110

Lang, M., Bischl, B., \& Surmann, D. (2017). batchtools: Tools for $\mathrm{R}$ to work on batch systems. Journal of Open Source Software, 2(10), 135. doi: $10.21105 /$ joss.00135

Langmeyer, A., Guglhör-Rudan, A., \& Tarnai, C. (2012). What Do Music Preferences Reveal About Personality? A Cross-Cultural Replication Using SelfRatings and Ratings of Music Samples. Journal of Individual Differences, 33(2), 119-130. doi:10.1027/1614-0001/a000082

Lathia, N., Sandstrom, G. M., Mascolo, C., \& Rentfrow, P. J. (2017). Happier People Live More Active Lives: Using Smartphones to Link Happiness and Physical Activity. PLOS ONE, 12(1), e0160589. doi:10.1371/journal.pone.0160589

Mahmoodi, J., Leckelt, M., Zalk, M. W. van, Geukes, K., \& Back, M. D. (2017, December). Big Data approaches in social and behavioral science: four key trade-offs and a call for integration. Elsevier. doi:10.1016/j.cobeha.2017.07.001

Masters, G. N. (1982). A rasch model for partial credit scoring. Psychometrika, 47(2), 149-174. doi:10.1007/BF02296272

Matz, S. C., Kosinski, M., Nave, G., \& Stillwell, D. J. (2017). Psychological targeting as an effective approach to digital mass persuasion. Proceedings of the National Academy of Sciences of the United States of America, 114(48), 12714-12719. doi:10.1073/pnas.1710966114

McCrae, R. R., \& John, O. P. (1992). An Introduction to the Five-Factor Model and Its Applications. Journal of Personality, 60(2), 175-215. doi:10.1111/j.14676494.1992.tb00970.x

McCrae, R. R., Costa, P., Jr, \& Martin, A., Thomas. (2005). The NEO-PI-3: A More Read- able Revised NEO Personality Inventory. Journal of Personality Assessment, 84(3), 261-270. doi:10.1207/s15327752jpa8403_05

Mehl, M. R., Gosling, S. D., \& Pennebaker, J. W. (2006). Personality in its natural habitat: manifestations and implicit folk theories of personality in daily life. Journal of Personality and Social Psychology, 90(5), 862-877. doi:10.1037/0022-3514.90.5.862

Mehrotra, A., Müller, S. R., Harari, G. M., Gosling, S. D., Mascolo, C., Musolesi, M., \& Rentfrow, P. J. (2017). Understanding the Role of Places and Activities on Mobile Phone Interaction and Usage Patterns. Proceedings of the ACM on Interactive, Mobile, Wearable and Ubiquitous Technologies, 1(3), 1-22. doi:10.1145/3131901

Miller, G. (2012). The Smartphone Psychology Manifesto. Perspectives on Psychological Science, 7(3), 221237. doi:10.1177/1745691612441215

Millgram, Y., Joormann, J., Huppert, J. D., \& Tamir, M. (2015). Sad as a Matter of Choice? Emotion-Regulation Goals in Depression. Psychological Science, 26(8), 1216-1228. doi:10.1177/0956797615583295

Mohammadi, G., \& Vinciarelli, A. (2012). Automatic Personality Perception: Prediction of Trait Attribution Based on Prosodic Features. IEEE Transactions on Affective Computing, 3(3), 273-284. doi:10.1109/TAFFC.2012.5

Molnar, C., Casalicchio, G., \& Bischl, B. (2018). iml: An R package for Interpretable Machine Learning. The Journal of Open Source Software, 26(3), 786. doi:10.21105/joss.00786

Montag, C., Blaszkiewicz, K., Lachmann, B., Andone, I., Sariyska, R., Trendafilov, B., ... Markowetz, A. (2014). Correlating Personality and Actual Phone Usage: Evidence From Psychoinformatics. Journal of Individual Differences, 35(3), 158-165. doi:10.1027/1614-0001/a000139

Montag, C., Blaszkiewicz, K., Sariyska, R., Lachmann, B., Andone, I., Trendafilov, B., ... Markowetz, A. (2015). Smartphone usage in the 21st century: who is active on WhatsApp? BMC Research Notes, 8(1), 331. doi:10.1186/s13104-015-1280-z

Mussel, P., Winter, C., Gelléri, P., \& Schuler, H. (2011). Explicating the openness to experience construct and its subdimensions and facets in a work setting. International Journal of Selection and Assessment, 19(2), 
145-156. doi:10.1111/j.1468-2389.2011.00542.x

Mønsted, B., Mollgaard, A., \& Mathiesen, J. (2018). Phonebased metric as a predictor for basic personality traits. Journal of Research in Personality, 74, 16-22. doi:10.1016/J.JRP.2017.12.004

Nave, G., Minxha, J., Greenberg, D. M., Kosinski, M., Stillwell, D., \& Rentfrow, P. J. (2018). Musical Preferences Predict Personality: Evidence From Active Listening and Facebook Likes. Psychological Science, 29(7), 1145-1158. doi:10.1177/0956797618761659

Ormel, J., Jeronimus, B. F., Kotov, R., Riese, H., Bos, E. H., Hankin, B., ... Oldehinkel, A. J. (2013). Neuroticism and common mental disorders: Meaning and utility of a complex relationship. Clinical Psychology Review, 33(5), 686-97. doi:10.1016/j.cpr.2013.04.003

Ozer, D. J., \& Benet-Martínez, V. (2006). Personality and the Prediction of Consequential Outcomes. Annual Review of Psychology, 57(1), 401-421. doi:10.1146/annurev.psych.57.102904.190127

Pargent, F., \& Albert-von der Gönna, J. (2018). Predictive Modeling With Psychological Panel Data. Zeitschrift Für Psychologie, 226(4), 246-258. doi: $10.1027 / 2151-2604 / \mathrm{a} 000343$

Park, G., Schwartz, H. A., Eichstaedt, J. C., Kern, M. L., Kosinski, M., Stillwell, D. J., ... Seligman, M. E. P. (2015). Automatic personality assessment through social media language. Journal of Personality and Social Psychology, 108(6). doi:10.1037/pspp0000020

Paunonen, S. V., \& Ashton, M. C. (2001). Big five factors and facets and the prediction of behavior. Journal of Personality and Social Psychology, 81(3), 524-539. doi:10.1037/0022-3514.81.3.524

Podsakoff, P. M., MacKenzie, S. B., \& Podsakoff, N. P. (2012). Sources of Method Bias in Social Science Research and Recommendations on How to Control It. Annual Review of Psychology, 63(1), 539-569. doi:10.1146/annurev-psych-120710-100452

Podsakoff, P. M., MacKenzie, S. B., Lee, J.-Y., \& Podsakoff, N. P. (2003). Common method biases in behavioral research: A critical review of the literature and recommended remedies. Journal of Applied Psychology, 88(5), 879-903. doi:10.1037/00219010.88.5.879

R Core Team. (2018). R: A language and environment for statistical computing. Vienna, Austria: R Foun- dation for Statistical Computing. Retrieved from https://www.R-project.org/

Randler, C., Baumann, V. P., \& Horzum, M. B. (2014). Morningness-eveningness, Big Five and the BIS/BAS inventory. Personality and Individual Differences, 66, 64-67. doi:10.1016/j.paid.2014.03.010

Roberts, B. W., Bogg, T., Walton, K. E., Chernyshenko, O. S., \& Stark, S. E. (2004). A lexical investigation of the lower-order structure of conscientiousness. Journal of Research in Personality, 38(2), 164-178. doi:10.1016/S0092-6566(03)00065-5

Roberts, B. W., Kuncel, N. R., Shiner, R., Caspi, A., \& Goldberg, L. R. (2007). The Power of Personality: The Comparative Validity of Personality Traits, Socioeconomic Status, ad Cognitive Ability for Predicting Important Life Outcomes. Perspectives on Psychological Science, 2(4), 313-45. doi:10.1111/j.17456916.2007.00047.x

Roenneberg, T., Wirz-Justice, A., \& Merrow, M. (2003). Life between clocks: Daily temporal patterns of human chronotypes. Journal of Biological Rhythms, 18(1), 80-90.

Roets, A., Cornelis, I., \& Van Hiel, A. (2014). Openness as a predictor of political orientation and conventional and unconventional political activism in western and eastern europe. Journal of Personality Assessment, 96(1), 53-63. doi:10.1080/00223891.2013.809354

Saarikallio, S., \& Erkkilä, J. (2007). The role of music in adolescents' mood regulation. Psychology of Music, 35(1), 88-109. doi:10.1177/0305735607068889

Saeb, S., Lattie, E. G., Schueller, S. M., Kording, K. P., \& Mohr, D. C. (2016). The relationship between mobile phone location sensor data and depressive symptom severity. PeerJ, 4, e2537. doi:10.7717/peerj.2537

Saeb, S., Zhang, M., Karr, C. J., Schueller, S. M., Corden, M. E., Kording, K. P., \& Mohr, D. C. (2015). Mobile Phone Sensor Correlates of Depressive Symptom Severity in Daily-Life Behavior: An Exploratory Study. Journal of Medical Internet Research, 17(7), e175. doi:10.2196/jmir.4273

Schoedel, R., Au, Q., Völkel, S. T., Lehmann, F., Becker, D., Bühner, M., ... Stachl, C. (2018). Digital Footprints of Sensation Seeking. Zeitschrift Für Psychologie, 226(4), 232-245. doi:10.1027/2151-2604/a000342

Schuwerk, T., Kaltefleiter, L., Au, Q., Hösl, A., \& Stachl, C. (2018, October). Enter the wild: Autistic traits and their relationship to mentalizing and 
social interaction in everyday life. PsyArXiv. doi:10.31234/osf.io/rxatn

Schwartz, H. A., Eichstaedt, J. C., Kern, M. L., Dziurzynski, L., Ramones, S. M., Agrawal, M., ... Ungar, L. H. (2013). Personality, Gender, and Age in the Language of Social Media: The OpenVocabulary Approach. PloS One, 8(9), e73791. doi:10.1371/journal.pone.0073791

Settanni, M., Azucar, D., \& Marengo, D. (2018). Predicting Individual Characteristics from Digital Traces on Social Media: A Meta-Analysis. Cyberpsychology, Behavior, and Social Networking, 21(4), 217-228. doi:10.1089/cyber.2017.0384

Skånland, M. S. (2013). Everyday music listening and affect regulation: The role of MP3 players. International Journal of Qualitative Studies on Health and WellBeing, 8(1), 20595. doi:10.3402/qhw.v8i0.20595

Spotify. (n.d.-a). Get a Track | Spotify for Developers. Retrieved from https://developer.spotify.com/ documentation/web-api/reference/tracks/get-track/

Spotify. (n.d.-b). Get Audio Features for a Track I Spotify for Developers. Retrieved from https://developer.spotify.com/documentation/ web-api/reference/tracks/get-audio-features/

Stachl, C., Au, Q., Schoedel, R., Buschek, D., Völkel, S., Schuwerk, T., ... al. (2019, June). Behavioral patterns in smartphone usage predict big five personality traits. OSF. doi:10.17605/OSF.IO/KQJHR

Stachl, C., Hilbert, S., Au, J.-Q., Buschek, D., De Luca, A., Bischl, B., ... Bühner, M. (2017). Personality Traits Predict Smartphone Usage. European Journal of Personality, 31(6), 701-722. doi:10.1002/per.2113

Stachl, C., Schoedel, R., Au, Q., Völkel, S., Buschek, D., Hussmann, H., ... Bühner, M. (2018, June). The PhoneStudy project. Open Science Framework. doi:10.17605/OSF.IO/UT42Y

Thoma, M. V., Ryf, S., Mohiyeddini, C., Ehlert, U., \& Nater, U. M. (2012). Emotion regulation through listening to music in everyday situations. Cognition E Emotion, 26(3), 550-560. doi:10.1080/02699931.2011.595390

Tsaousis, I. (2010). Circadian preferences and personality traits: A meta-analysis. European Journal of Personality: Published for the European Association of Personality Psychology, 24(4), 356-373. doi:10.1002/per.754

Union, I. T. (2018). Measuring the Information Society Report 2018. ITU Publications, 1, 2-18. Retrieved from https://www.itu.int/en/ITU-D/Statistics/Pages/ publications/misr2018.aspx

Vaerenbergh, Y. V., \& Thomas, T. D. (2013). Response Styles in Survey Research: A Literature Review of Antecedents, Consequences, and Remedies. International Journal of Public Opinion Research, 25(2), 195-217. doi:10.1093/ijpor/eds021

Whiteside, S. P., \& Lynam, D. R. (2001). The Five Factor Model and impulsivity: using a structural model of personality to understand impulsivity. Personality and Individual Differences, 30(4), 669-689. doi:10.1016/S0191-8869(00)00064-7

Wilson, R. E., Gosling, S. D., \& Graham, L. T. (2012). A Review of Facebook Research in the Social Sciences. Perspectives on Psychological Science : A Journal of the Association for Psychological Science, 7(3), 203-220. doi:10.1177/1745691612442904

Wright, M. N., \& Ziegler, A. (2017). ranger: A fast implementation of random forests for high dimensional data in C++ and R. Journal of Statistical Software, 77(1), 1-17. doi:10.18637/jss.v077.i01

Wrzus, C., \& Mehl, M. R. (2015). Lab and/or Field? Measuring Personality Processes and Their Social Consequences. European Journal of Personality, 29(2), 250-271. doi:10.1002/per.1986

Yarkoni, T. (2010). Personality in 100,000 Words: A largescale analysis of personality and word use among bloggers. Journal of Research in Personality, 44(3), 363-373. doi:10.1016/j.jrp.2010.04.001

Yarkoni, T., \& Westfall, J. (2017). Choosing Prediction Over Explanation in Psychology: Lessons From Machine Learning. Perspectives on Psychological Science, 12(6), 1100-1122. doi:10.1177/1745691617693393

Youyou, W., Kosinski, M., \& Stillwell, D. (2015). Computerbased personality judgments are more accurate than those made by humans. Proceedings of the National Academy of Sciences of the United States of America, 112(4), 1036-40. doi:10.1073/pnas.1418680112

Ziegler, M., Bensch, D., Maaß, U., Schult, V., Vogel, M., \& Bühner, M. (2014). Big Five facets as predictor of job training performance: The role of specific job demands. Learning and Individual Differences, 29, 1-7. doi:10.1016/j.lindif.2013.10.008

Zou, H., \& Hastie, T. (2005). Regularization and variable selection via the elastic net. Journal of the Royal Statistical Society. Series B: Statistical Methodology, 67(2), 301-320. doi:10.1111/j.14679868.2005.00503.x 